\title{
Fourier multipliers for Hölder continuous functions and maximal regularity
}

\author{
by \\ Wolfgang Arendt (Ulm), Charles Batty (Oxford) \\ and Shangquan Bu (Beijing and Ulm)
}

\begin{abstract}
Two operator-valued Fourier multiplier theorems for Hölder spaces are proved, one periodic, the other on the line. In contrast to the $L^{p}$-situation they hold for arbitrary Banach spaces. As a consequence, maximal regularity in the sense of Hölder can be characterized by simple resolvent estimates of the underlying operator.
\end{abstract}

1. Introduction. The aim of this article is to characterize wellposedness of linear differential equations with maximal regularity in Hölder spaces. More specifically, we consider the following two problems, the first with periodic boundary conditions, the second on the real line.

Let $A$ be a closed operator on a Banach space $X$ and let $\alpha \in(0,1)$. By $C_{\mathrm{per}}^{\alpha}([0,2 \pi], X)$ we denote the space of all Hölder continuous functions $f:[0,2 \pi] \rightarrow X$ of Hölder index $\alpha$ such that $f(0)=f(2 \pi)$. We say that the problem

$\left(\mathrm{P}_{\mathrm{per}}\right)$

$$
\left\{\begin{array}{l}
u^{\prime}(t)=A u(t)+f(t) \quad(t \in[0,2 \pi]), \\
u(0)=u(2 \pi)
\end{array}\right.
$$

is $C^{\alpha}$-well-posed if, for each $f \in C_{\text {per }}^{\alpha}([0,2 \pi], X)$, there is a unique solution $u \in C_{\mathrm{per}}^{1+\alpha}([0,2 \pi], X) \cap C_{\mathrm{per}}^{\alpha}([0,2 \pi], D(A))$. This means that there exists a classical solution with maximal regularity.

It is remarkable that well-posedness can be characterized completely in terms of the resolvent of $A$ without any restriction on the Banach space $X$. In fact, we show in Theorem 4.2 that the problem $\left(\mathrm{P}_{\text {per }}\right)$ is $C^{\alpha}$-well-posed if and only if $i k-A$ is invertible for all $k \in \mathbb{Z}$ and $\sup _{k \in \mathbb{Z}}\left\|k(i k-A)^{-1}\right\|<\infty$.

On the real line we show the following analogous result (Theorem 6.1).

2000 Mathematics Subject Classification: Primary 42A45; Secondary 34G10, 47D06.

This research is part of the DFG-project: "Regularität und Asymptotik für elliptische und parabolische Probleme". The third author is supported by the Alexander-vonHumboldt Foundation and the NSF of China. The authors are very grateful to Matthias Hieber, André Noll, Maria Girardi and Lutz Weis for helpful discussions and for providing a preprint of $[\mathrm{GW}]$. 
For each $f \in C^{\alpha}(\mathbb{R}, X)$ there exists a unique $u \in C^{1+\alpha}(\mathbb{R}, X) \cap C^{\alpha}(\mathbb{R}, D(A))$ solving the problem

$$
u^{\prime}(t)=A u(t)+f(t) \quad(t \in \mathbb{R})
$$

if and only if $i s-A$ is invertible for each $s \in \mathbb{R}$ and $\sup _{s \in \mathbb{R}}\left\|s(i s-A)^{-1}\right\|<\infty$.

Our main tools are two operator-valued Fourier multiplier theorems, a periodic one proved in Section 3 and one on the real line proved in Section 5. These multiplier theorems are obtained as consequences of a basic estimate for convolution with smooth operator-valued functions with compact support given in Section 2. This estimate involves a norm of Mikhlin's type on the multiplier, and such estimates are well known in the scalar case (see [Hö, Theorem 7.9.6] and [Tr, Sections 2.3.7 and 5.2.2]). On the line (but not in the periodic case), similar operator-valued multiplier theorems for Hölder spaces, and more generally Besov spaces, have been obtained by Amann [Am] (see also the research announcement by Weis [We1], as well as the recent manuscript by Girardi and Weis [GW]). However, they differ from ours in several aspects. We consider arbitrary Hölder continuous functions and not only bounded functions in the Hölder space. Accordingly, we prove an estimate for the homogeneous Mikhlin norm (in contrast to the inhomogeneous norm considered in $[\mathrm{Am}]$, [We1] and [GW]). It is possible to deduce the homogeneous estimate from the inhomogeneous one by scaling and approximation, but we give a direct and completely elementary method using pointwise estimates on Fourier transforms. This avoids any use of dyadic decompositions in the Fourier image, the theory of vector-valued distributions or duality of function spaces which occur in approaches via Besov spaces.

Our characterization of $C^{\alpha}$-maximal regularity of the problems $(\mathrm{P})$, $\left(\mathrm{P}_{\text {per }}\right)$ and some second order problems treated in Section 4 is new to our knowledge. But some of the well-known estimates for generators of holomorphic semigroups (see the monograph of Lunardi [Lu]) can be deduced from it.

More is known about maximal regularity of problems $\left(\mathrm{P}_{\text {per }}\right)$ and $(\mathrm{P})$ in the $L^{p}$-sense. Mielke [Mi] was the first to consider problem $(\mathrm{P})$ on Hilbert spaces and he actually characterized $L^{p}$-maximal regularity in terms of the resolvent. It was Weis [We2] who discovered the right condition on a class of Banach spaces (UMD-spaces), after previous work by Clément, de Pagter, Sukochev and Witvliet [CPSW]. Thus maximal regularity of the inhomogenous equation with a Dirichlet boundary condition on a UMD-space can be characterized by $R$-boundedness of the resolvent (see the papers of Weis [We2] and Clément and Prüss [CP]; see also [AB3]). A basic theorem in this context is the operator-valued version of Mikhlin's multiplier theorem due to Weis [We2], where the Mikhlin estimate is formulated in terms of $R$-boundedness. It allows one in particular to characterize maximal regu- 
larity of problem $(\mathrm{P})$ in the sense of $L^{p}$ (see the thesis of Schweiker [Sch]). A periodic operator-valued multiplier theorem for $L^{p}$-spaces is given in [AB1] where $L^{p}$-maximal regularity of $\left(\mathrm{P}_{\mathrm{per}}\right)$ is characterized. However, in contrast to the $C^{\alpha}$-multiplier theorems given here, each of the $L^{p}$-multiplier theorems (the one by Weis on the line as well as the periodic one) holds in the classical formulation (involving merely boundedness instead of $R$-boundedness) if and only if the underlying Banach space is isomorphic to a Hilbert space (see [AB1] and [AB2]). It was discovered by Amann [Am] (see also [We1] and $[\mathrm{GW}])$ that multipliers on Hölder spaces behave better in this respect.

2. The basic estimate. Throughout, $X, Y$ will be Banach spaces, and $\mathcal{L}(X, Y)$ (or $\mathcal{L}(X)$ when $Y=X)$ will be the space of all bounded linear operators from $X$ to $Y$.

Let $0<\alpha<1$. By $C^{\alpha}(\mathbb{R}, X)$ we denote the space of all Hölder continuous functions $f: \mathbb{R} \rightarrow X$ of exponent $\alpha$. There is a seminorm $\|\cdot\|_{\alpha}$ on $C^{\alpha}(\mathbb{R}, X)$ given by

$$
\|f\|_{\alpha}=\sup _{s \neq t} \frac{\|f(s)-f(t)\|}{|s-t|^{\alpha}} .
$$

Moreover, $C^{\alpha}(\mathbb{R}, X)$ is a Banach space for the norm

$$
\|f\|_{C^{\alpha}}:=\|f\|_{\alpha}+\|f(0)\| \text {. }
$$

Since

$$
\|f(t)\| \leq\|f(0)\|+\|f\|_{\alpha}|t|^{\alpha} \quad(t \in \mathbb{R}),
$$

convergence with respect to the norm $\|\cdot\|_{C^{\alpha}}$ implies uniform convergence on compact intervals.

Let $M \in C^{2}(\mathbb{R} \backslash\{0\}, \mathcal{L}(X, Y))$. We define the Mikhlin norm of order 2 by

$$
\|M\|_{\mathcal{M}}=\sup _{t \neq 0}\|M(t)\|+\sup _{t \neq 0}\left\|t M^{\prime}(t)\right\|+\sup _{t \neq 0}\left\|t^{2} M^{\prime \prime}(t)\right\| .
$$

The spaces $\mathcal{M}(\mathbb{R}, \mathcal{L}(X, Y))$ and $\mathcal{M}(\mathbb{R} \backslash\{0\}, \mathcal{L}(X, Y))$ consist of those functions $M \in C^{2}(\mathbb{R}, \mathcal{L}(X, Y))$ (resp., $\left.M \in C^{2}(\mathbb{R} \backslash\{0\}, \mathcal{L}(X, Y))\right)$ such that $\|M\|_{\mathcal{M}}<\infty$.

By $C_{\mathrm{c}}^{2}(\mathbb{R}, \mathcal{L}(X, Y))\left(\right.$ resp., $C_{\mathrm{c}}^{2}(\mathbb{R} \backslash\{0\}, \mathcal{L}(X, Y))$ we denote the space of all functions $M \in C^{2}(\mathbb{R}, \mathcal{L}(X, Y))$ with compact support supp $M$ (such that $0 \notin \operatorname{supp} M$, respectively). For $M \in C_{\mathrm{c}}^{2}(\mathbb{R}, \mathcal{L}(X, Y))$, the Fourier transform $\mathcal{F} M$ of $M$ is given by

$$
(\mathcal{F} M)(s)=\int_{\mathbb{R}} e^{-i t s} M(t) d t .
$$

Note that $s^{2}(\mathcal{F} M)(s)=-\left(\mathcal{F} M^{\prime \prime}\right)(s)$. Hence

$$
\|(\mathcal{F} M)(s)\| \leq \max \left(\|M\|_{L^{1}},\left\|M^{\prime \prime}\right\|_{L^{1}} s^{-2}\right) .
$$


If $f \in C^{\alpha}(\mathbb{R}, X)$, it follows from (2.2) and (2.3) that

$$
\int_{\mathbb{R}}\|(\mathcal{F} M)(s)\|\|f(t-s)\| d s \leq c\left(1+|t|^{\alpha}\right)
$$

for some constant $c$ (depending on $\alpha, f$ and $M$ ). Hence the convolution integral

$$
(\mathcal{F} M * f)(t):=\int_{\mathbb{R}}(\mathcal{F} M)(t-s) f(s) d s=\int_{\mathbb{R}}(\mathcal{F} M)(s) f(t-s) d s
$$

is well defined for all $t \in \mathbb{R}$.

The aim of this section is to prove the following basic estimate.

Proposition 2.1. For each $\alpha \in(0,1)$ there exists a constant $c_{\alpha}>0$ such that

$$
\|\mathcal{F} M * f\|_{\alpha} \leq c_{\alpha}\|M\|_{\mathcal{M}}\|f\|_{\alpha}
$$

for all $f \in C^{\alpha}(\mathbb{R}, X)$ and all $M \in C_{\mathrm{c}}^{2}(\mathbb{R}, \mathcal{L}(X, Y))$.

This estimate will enable us to define Fourier multipliers associated with $M \in \mathcal{M}(\mathbb{R} \backslash\{0\}, \mathcal{L}(X, Y)$ ) in Section 5 (and the periodic analogue in Section 3) by taking an approximating sequence $\left(M_{n}\right)_{n \in \mathbb{N}}$ in $C_{\mathrm{c}}^{2}(\mathbb{R}, \mathcal{L}(X, Y))$. The existence of suitable $M_{n}$ is a consequence of the following splitting result (more precisely, of Corollary 2.3), which will be used several times in the construction.

Lemma 2.2. There is a constant $\kappa>0$ such that the following holds. Given $a>0$ and $M \in \mathcal{M}(\mathbb{R} \backslash\{0\}, \mathcal{L}(X, Y))$ there exist $M_{1}, M_{2} \in \mathcal{M}(\mathbb{R} \backslash\{0\}$, $\mathcal{L}(X, Y))$ such that $M=M_{1}+M_{2}, \operatorname{supp} M_{1} \subset[-2 a, 2 a] \cap \operatorname{supp} M, \operatorname{supp} M_{2}$ $\subset((-\infty,-a] \cup[a, \infty)) \cap \operatorname{supp} M$, and $\left\|M_{j}\right\|_{\mathcal{M}} \leq \kappa\|M\|_{\mathcal{M}}(j=1,2)$.

Proof. Let $\Phi: \mathbb{R} \rightarrow[0,1]$ be a $C^{2}$-function such that $\Phi(t)=1(t \in$ $[-1,1])$ and $\operatorname{supp} \Phi \subset[-2,2]$. Let

$$
M_{1}(t)=\Phi(t / a) M(t), \quad M_{2}(t)=(1-\Phi(t / a)) M(t) .
$$

We have to check that $\left\|M_{j}\right\|_{\mathcal{M}} \leq \kappa\|M\|_{\mathcal{M}}$. Clearly, $\left\|M_{1}(t)\right\| \leq\|M(t)\| \leq$ $\|M\|_{\mathcal{M}}(t \in \mathbb{R} \backslash\{0\})$. Furthermore

$$
t M_{1}^{\prime}(t)= \begin{cases}(t / a) \Phi^{\prime}(t / a) M(t)+\Phi(t / a) t M^{\prime}(t) & \text { if } a \leq|t| \leq 2 a \\ \Phi(t / a) t M^{\prime}(t) & \text { otherwise }\end{cases}
$$

Hence

$$
\left\|t M_{1}^{\prime}(t)\right\| \leq 2\left\|\Phi^{\prime}\right\|_{\infty}\|M(t)\|+\left\|t M^{\prime}(t)\right\| \leq\left(2\left\|\Phi^{\prime}\right\|_{\infty}+1\right)\|M\|_{\mathcal{M}} .
$$

Also

$$
t^{2} M_{1}^{\prime \prime}(t)= \begin{cases}(t / a)^{2} \Phi^{\prime \prime}(t / a) M(t)+2(t / a) \Phi^{\prime}(t / a) t M^{\prime}(t)+\Phi(t / a) t^{2} M^{\prime \prime}(t) \\ \Phi(t / a) t^{2} M^{\prime \prime}(t) & \text { otherwise. }\end{cases}
$$


Hence

$$
\begin{aligned}
\left\|t^{2} M_{1}^{\prime \prime}(t)\right\| & \leq 4\left\|\Phi^{\prime \prime}\right\|_{\infty}\|M(t)\|+4\left\|\Phi^{\prime}\right\|_{\infty}\left\|t M^{\prime}(t)\right\|+\left\|t^{2} M^{\prime \prime}(t)\right\| \\
& \leq\left(4\left\|\Phi^{\prime \prime}\right\|_{\infty}+4\left\|\Phi^{\prime}\right\|_{\infty}+1\right)\|M\|_{\mathcal{M}} .
\end{aligned}
$$

This establishes the required properties of $M_{1}$. Those of $M_{2}$ follow since $M=M_{1}+M_{2}$.

Corollary 2.3. Let $M \in \mathcal{M}(\mathbb{R} \backslash\{0\}, \mathcal{L}(X, Y))$ and $0<a<b$. There exists $\widetilde{M} \in C_{\mathrm{c}}^{2}(\mathbb{R}, \mathcal{L}(X, Y))$ such that $\widetilde{M}(t)=M(t)$ whenever $a<|t|<b$, supp $\widetilde{M} \subset[-2 b,-a / 2] \cup[a / 2,2 b]$ and $\|\widetilde{M}\|_{\mathcal{M}} \leq \kappa^{2}\|M\|_{\mathcal{M}}$.

Proof. By Lemma 2.2, there exists $M_{1} \in \mathcal{M}(\mathbb{R} \backslash\{0\}, \mathcal{L}(X, Y))$ such that $M_{1}(t)=M(t)$ whenever $|t|<b, M_{1}(t)=0$ whenever $|t|>2 b$, and $\left\|M_{1}\right\|_{\mathcal{M}} \leq$ $\kappa\|M\|_{\mathcal{M}}$. Now by Lemma 2.2 again, there exists $\widetilde{M} \in \mathcal{M}(\mathbb{R} \backslash\{0\}, \mathcal{L}(X, Y))$ such that $\widetilde{M}(t)=M_{1}(t)$ whenever $|t|>a, \widetilde{M}(t)=0$ whenever $|t|<a / 2$, and $\|\widetilde{M}\|_{\mathcal{M}} \leq \kappa\left\|M_{1}\right\|_{\mathcal{M}}$. Now $\widetilde{M}$ has the required properties.

Now we turn more directly towards the proof of Proposition 2.1. There are several possible approaches (see Remarks 2.7) and we wish to present one which is very elementary. We therefore assume that $M \in C_{\mathrm{c}}^{2}(\mathbb{R}, \mathcal{L}(X, Y))$ and we first obtain some very simple pointwise estimates on $\mathcal{F} M$ and its first derivative.

Lemma 2.4. Let $M \in C_{\mathrm{c}}^{2}(\mathbb{R}, \mathcal{L}(X, Y))$ and $0<a<b$.

(1) If $\operatorname{supp} M \subset[-a, a]$, then $\left\|(\mathcal{F} M)^{\prime}(s)\right\| \leq a^{2}\|M\|_{\mathcal{M}}$ for all $s \in \mathbb{R}$.

(2) If $\operatorname{supp} M \subset[-b,-a] \cup[a, b]$, then

(a) $\left\|(\mathcal{F} M)^{\prime}(s)\right\| \leq 4\|M\|_{\mathcal{M}} \log (b / a) / s^{2}$,

(b) $\|(\mathcal{F} M)(s)\| \leq 2\|M\|_{\mathcal{M}} \log (b / a) /|s|$,

for all $s \in \mathbb{R} \backslash\{0\}$.

(3) If $\operatorname{supp} M \subset(-\infty,-b] \cup[b, \infty)$, then $\|(\mathcal{F} M)(s)\| \leq 2\|M\|_{\mathcal{M}} /\left(b s^{2}\right)$ for all $s \in \mathbb{R} \backslash\{0\}$.

Proof. Let $M_{1}(t)=t M(t)$, so $(\mathcal{F} M)^{\prime}(s)=-i\left(\mathcal{F} M_{1}\right)(s)$.

(1) In this case,

$$
\begin{aligned}
\left\|(\mathcal{F} M)^{\prime}(s)\right\| & \leq \int_{\mathbb{R}}\left\|M_{1}(t)\right\| d t \\
& =\int_{-a}^{a}|t|\|M(t)\| d t \leq a^{2} \sup _{t}\|M(t)\| \leq a^{2}\|M\|_{\mathcal{M}} .
\end{aligned}
$$


(2) In this case,

$$
\begin{aligned}
\left\|s^{2}(\mathcal{F} M)^{\prime}(s)\right\| & =\left\|i\left(\mathcal{F} M_{1}^{\prime \prime}\right)(s)\right\| \leq \int_{\mathbb{R}}\left\|t M^{\prime \prime}(t)+2 M^{\prime}(t)\right\| d t \\
& \leq 2 \int_{a}^{b} \frac{2\|M\|_{\mathcal{M}}}{t} d t=4\|M\|_{\mathcal{M}} \log (b / a), \\
\|s(\mathcal{F} M)(s)\| & =\left\|i\left(\mathcal{F} M^{\prime}\right)(s)\right\| \leq \int_{\mathbb{R}}\left\|M^{\prime}(t)\right\| d t \\
& \leq 2 \int_{a}^{b} \frac{\|M\|_{\mathcal{M}}}{t} d t=2\|M\|_{\mathcal{M}} \log (b / a) .
\end{aligned}
$$

(3) In this case,

$$
\begin{aligned}
\left\|s^{2}(\mathcal{F} M)(s)\right\| & =\left\|-\left(\mathcal{F} M^{\prime \prime}\right)(s)\right\| \leq \int_{\mathbb{R}}\left\|M^{\prime \prime}(t)\right\| d t \\
& \leq 2 \int_{b}^{\infty} \frac{\|M\|_{\mathcal{M}}}{t^{2}} d t=\frac{2}{b}\|M\|_{\mathcal{M}} .
\end{aligned}
$$

Corollary 2.5. Let $M \in C_{\mathrm{c}}^{2}(\mathbb{R}, \mathcal{L}(X, Y))$ and $a>0$.

(1) If $\operatorname{supp} M \subset[-a, a]$, then

$$
\left\|(\mathcal{F} M)^{\prime}(s)\right\| \leq \frac{4 \kappa \mid M \|_{\mathcal{M}}(1+\log (a|s|))}{s^{2}}
$$

whenever $|s| \geq 1 / a$.

(2) If $\operatorname{supp} M \subset(-\infty, a] \cup[a, \infty)$, then

$$
\|(\mathcal{F} M)(s)\| \leq \frac{4 \kappa\|M\|_{\mathcal{M}}(1+\log (1 / a|s|))}{|s|}
$$

whenever $|s| \leq 1 / a$.

Proof. (1) Fix $s$ with $|s| \geq 1 / a$. By Lemma 2.2, $M=M_{1}+M_{2}$ where $\operatorname{supp} M_{1} \subset[-2 /|s|, 2 /|s|], \operatorname{supp} M_{2} \subset[-a,-1 /|s|] \cup[1 /|s|, a]$ and $\left\|M_{j}\right\|_{\mathcal{M}} \leq$ $\kappa\|M\|_{\mathcal{M}}$. Then Lemma 2.4, (1) and (2)(a), give

$$
\begin{aligned}
\left\|\left(\mathcal{F} M_{1}\right)^{\prime}(s)\right\| & \leq \kappa\|M\|_{\mathcal{M}} 4 / s^{2}, \\
\left\|\left(\mathcal{F} M_{2}\right)^{\prime}(s)\right\| & \leq 4 \kappa\|M\|_{\mathcal{M}} \log (a|s|) / s^{2} .
\end{aligned}
$$

The claim follows.

(2) Fix $s$ with $|s| \leq 1 / a$. By Lemma 2.2, $M=M_{1}+M_{2}$ where supp $M_{1}$ $\subset[-1 /|s|,-a] \cup[a, 1 /|s|], \operatorname{supp} M_{2} \subset(-\infty,-1 /(2|s|)] \cup[1 /(2|s|), \infty)$ and 
$\left\|M_{j}\right\|_{\mathcal{M}} \leq \kappa\|M\|_{\mathcal{M}}$. Then by Lemma 2.4, (2)(b) and (3), we have

$$
\begin{aligned}
& \left\|\left(\mathcal{F} M_{1}\right)(s)\right\| \leq \frac{2 \kappa\|M\|_{\mathcal{M}} \log (1 / a|s|)}{|s|}, \\
& \left\|\left(\mathcal{F} M_{2}\right)(s)\right\| \leq \frac{2 \kappa\|M\|_{\mathcal{M}}}{s^{2} /(2|s|)}=\frac{4 \kappa\|M\|_{\mathcal{M}}}{|s|} .
\end{aligned}
$$

Now we are able to prove Proposition 2.1.

Proof of Proposition 2.1. Take $a>0$, and let $\kappa, M_{1}, M_{2}$ be as in Lemma 2.2. Then

$$
\int_{\mathbb{R}}\left(\mathcal{F} M_{2}\right)(s) d s=2 \pi M_{2}(0)=0
$$

Hence,

$$
\begin{aligned}
\left\|\left(\mathcal{F} M_{2} * f\right)(t)\right\| \\
=\left\|\int_{\mathbb{R}}\left(\mathcal{F} M_{2}\right)(s)(f(t-s)-f(t)) d s\right\| \\
\leq \int_{\mathbb{R}}\left\|\left(\mathcal{F} M_{2}\right)(s)\right\||s|^{\alpha}\|f\|_{\alpha} d s \\
\leq 2 \int_{0}^{1 / a} 4 \kappa\left\|M_{2}\right\|_{\mathcal{M}}(1+\log (1 / a s)) s^{\alpha-1}\|f\|_{\alpha} d s \\
\quad+2 \int_{1 / a}^{\infty} \frac{2\left\|M_{2}\right\|_{\mathcal{M}}}{a s^{2-\alpha}}\|f\|_{\alpha} d s \\
\leq\left(8 \kappa \int_{0}^{1}(1+\log (1 / s)) s^{\alpha-1} d s+4 \int_{1}^{\infty} \frac{d s}{s^{2-\alpha}}\right) a^{-\alpha} \kappa\|M\|_{\mathcal{M}}\|f\|_{\alpha} \\
=: c_{\alpha 2} a^{-\alpha}\|M\|_{\mathcal{M}}\|f\|_{\alpha},
\end{aligned}
$$

where we have used Corollary 2.5 and Lemma $2.4(3)$ to estimate $\left\|\left(\mathcal{F} M_{2}\right)(s)\right\|$ for $|s| \leq 1 / a$ and $|s| \geq 1 / a$ respectively. Moreover,

$$
\int_{\mathbb{R}}\left(\mathcal{F} M_{1}\right)^{\prime}(s) d s=\lim _{b \rightarrow \infty}\left(\left(\mathcal{F} M_{1}\right)(b)-\left(\mathcal{F} M_{1}\right)(-b)\right)=0 .
$$

Hence,

$$
\begin{aligned}
\left\|\left(\mathcal{F} M_{1} * f\right)^{\prime}(t)\right\| & =\left\|\int_{\mathbb{R}}\left(\mathcal{F} M_{1}\right)^{\prime}(s)(f(t-s)-f(t)) d s\right\| \\
& \leq \int_{\mathbb{R}}\left\|\left(\mathcal{F} M_{1}\right)^{\prime}(s)\right\||s|^{\alpha}\|f\|_{\alpha} d s
\end{aligned}
$$




$$
\begin{aligned}
& \leq 2 \int_{0}^{1 / a}\left\|M_{1}\right\|_{\mathcal{M}} a^{2} s^{\alpha}\|f\|_{\alpha} d s \\
& \quad+2 \int_{1 / a}^{\infty} 4 \kappa\left\|M_{1}\right\|_{\mathcal{M}}(1+\log (a s)) s^{\alpha-2}\|f\|_{\alpha} d s \\
& \leq\left(2 \int_{0}^{1} s^{\alpha} d s+8 \kappa \int_{1}^{\infty}(1+\log s) s^{\alpha-2} d s\right) a^{1-\alpha} \kappa\|M\|_{\mathcal{M}}\left\|_{f}\right\|_{\alpha} \\
& =: c_{\alpha 1} a^{1-\alpha}\|M\|_{\mathcal{M}}\|f\|_{\alpha},
\end{aligned}
$$

where we have used Lemma 2.4(1) for $|s| \leq 1 / a$ and Corollary 2.5 for $|s|$ $\geq 1 / a$.

Now take $t_{1} \neq t_{2}$. Then

$$
\begin{aligned}
\left\|(\mathcal{F} M * f)\left(t_{1}\right)-(\mathcal{F} M * f)\left(t_{2}\right)\right\| \\
\quad \leq\left\|\int_{t_{1}}^{t_{2}}\left(\mathcal{F} M_{1} * f\right)^{\prime}(t) d t\right\|+\left\|\left(\mathcal{F} M_{2} * f\right)\left(t_{1}\right)\right\|+\left\|\left(\mathcal{F} M_{2} * f\right)\left(t_{2}\right)\right\| \\
\leq\left(\left|t_{2}-t_{1}\right| c_{\alpha 1} a^{1-\alpha}+2 c_{\alpha 2} a^{-\alpha}\right)\|M\|_{\mathcal{M}}\|f\|_{\alpha} \\
\leq \frac{c_{\alpha}}{3}\left(\left|t_{2}-t_{1}\right| a^{1-\alpha}+2 a^{-\alpha}\right)\|M\|_{\mathcal{M}}\left\|_{f}\right\|_{\alpha},
\end{aligned}
$$

where $c_{\alpha}=3 \max \left\{c_{\alpha 1}, c_{\alpha 2}\right\}$. This is valid for all $a>0$, and choosing $a=$ $\left|t_{1}-t_{2}\right|^{-1}$ gives $\left\|(\mathcal{F} M * f)\left(t_{1}\right)-(\mathcal{F} M * f)\left(t_{2}\right)\right\| \leq c_{\alpha}\|M\|_{\mathcal{M}}\|f\|_{\alpha}\left|t_{1}-t_{2}\right|^{\alpha}$.

REMARK 2.6 (bounded Hölder continuous functions). For an operatorvalued function $M \in C_{\mathrm{c}}^{2}(\mathbb{R}, \mathcal{L}(X, Y))$ we can also consider the inhomogeneous Mikhlin norm of order 2 :

$$
\|M\| \tilde{\mathcal{M}}:=\sup _{t}\|M(t)\|+\sup _{t}(1+|t|)\left\|M^{\prime}(t)\right\|+\sup _{t}\left(1+t^{2}\right)\left\|M^{\prime \prime}(t)\right\| .
$$

Suppose that $f \in C^{\alpha}(\mathbb{R}, X)$ is bounded. Then

$$
\|\mathcal{F} M * f\|_{\alpha}+\|\mathcal{F} M * f\|_{\infty} \leq \text { const } \cdot\|M\| \tilde{\mathcal{M}}\left(\|f\|_{\alpha}+\|f\|_{\infty}\right) .
$$

In fact, fixing $a=1$ and taking $M_{1}=\Phi M$ as in Lemma 2.2, one has $\left\|M_{1}\right\|_{L^{1}}=\int_{-2}^{2}\left|M_{1}(s)\right| d s \leq 4\|M\|_{\infty} \leq 4\|M\| \tilde{\mathcal{M}}$ and $\left\|M_{1}^{\prime \prime}\right\|_{L^{1}}=\int_{-2}^{2}\left|M_{1}^{\prime \prime}(s)\right| d s$ $\leq$ const $\cdot\|M\| \tilde{\mathcal{M}}$. It follows from $(2.3)$ that

$$
\left\|\left(\mathcal{F} M_{1} * f\right)(t)\right\| \leq \int_{\mathbb{R}}\left\|\left(\mathcal{F} M_{1}\right)(s)\right\|\|f\|_{\infty} d s \leq \text { const } \cdot\|M\|_{\mathcal{M}}\|f\|_{\infty} .
$$

Combining this with (2.6) above, (2.7) follows.

Remarks 2.7. (a) The estimate (2.7) has been proven by Amann [Am] (see also [We1] and [GW]) as a special case of a result about Fourier multipliers on Besov spaces. In the scalar case an estimate of this type can be 
found in the monograph of Triebel [Tr, Section 2.3.7]. Their proofs depend on dyadic decomposition, the theory of distributions and duality. It is possible to deduce Proposition 2.1 from (2.7) by scaling and approximation (see the proof of Proposition 2.8 below).

(b) A. Noll (private communication) has proved the basic estimate (2.5) by using a dyadic decomposition as in [Hö, Theorem 7.9.6].

Recall that a Banach space $X$ has Fourier type $p$, where $1 \leq p \leq 2$, if the Fourier transform defines a bounded linear operator from $L^{p}(\mathbb{R}, X)$ to $L^{p^{\prime}}(\mathbb{R}, X)$, where $p^{\prime}$ is the conjugate index of $p$. For example, a space of the form $L^{p}(\Omega)$, where $1 \leq p \leq 2$, has Fourier type $p$; $X$ has Fourier type 2 if and only if $X$ is a Hilbert space; $X$ has Fourier type $p$ if and only if $X^{*}$ has Fourier type $p$. Every Banach space has Fourer type 1; $X$ is $B$-convex if it has Fourier type $p$ for some $p>1$. Every uniformly convex space is $B$-convex.

Assume that $X$ and $Y$ are $B$-convex. Then Girardi and Weis [GW] have shown that (2.7) remains valid if the norm $\|M\| \tilde{\mathcal{M}}$ is replaced by the inhomogeneous Mikhlin norm of order 1, i.e., by

$$
\|M\|_{\mathcal{M}_{1}}:=\sup _{t}\|M(t)\|+\sup _{t}(1+|t|)\left\|M^{\prime}(t)\right\| .
$$

We shall now show that if $X$ is $B$-convex, the estimate (2.5) of Proposition 2.1 can be improved by replacing $\|M\|_{\mathcal{M}}$ by the (homogeneous) Mikhlin norm of order 1 , i.e., by

$$
\|M\|_{\mathcal{M}_{1}}:=\sup _{t}\|M(t)\|+\sup _{t}\left\|t M^{\prime}(t)\right\|
$$

We shall deduce this from the result of $[\mathrm{GW}]$ by means of a scaling argument. The proof in $[\mathrm{GW}]$ is set in the context of Besov spaces and uses a dyadic decomposition in a way which seems to be unavoidable. So far, it seems not to be known whether the geometric condition on $X$ can be omitted.

Proposition 2.8. Let $X$ be a $B$-convex space and $Y$ be arbitrary. For each $\alpha \in(0,1)$ there exists a constant $c_{\alpha, X}>0$ (depending only on $\alpha$ and $X)$ such that

$$
\|\mathcal{F} M * f\|_{\alpha} \leq c_{\alpha, X}\|M\|_{\mathcal{M}_{1}}\|f\|_{\alpha}
$$

for all $f \in C^{\alpha}(\mathbb{R}, X)$ and all $M \in C_{\mathrm{c}}^{2}(\mathbb{R}, \mathcal{L}(X, Y))$.

Proof. First, assume that $Y=\mathbb{C}$. The Besov space $B_{\infty, \infty}^{\alpha}(\mathbb{R}, X)$ is the space of all bounded functions $f \in C^{\alpha}(\mathbb{R}, X)$, and the Besov norm is equivalent to the norm $\|f\|_{\alpha}+\|f\|_{\infty}([\operatorname{Tr}$, Theorem 2.5.7], [Am, p. 25]). Hence it follows from [GW, Theorem 4.7 and Corollary 4.10] that there is a constant $c$ (depending only on $\alpha$ and $X$ ) such that

$$
\|\mathcal{F} M * f\|_{\alpha}+\|\mathcal{F} M * f\|_{\infty} \leq c\|M\| \tilde{\mathcal{M}}_{1}\left(\|f\|_{\alpha}+\|f\|_{\infty}\right)
$$

whenever $f \in C^{\alpha}(\mathbb{R}, X)$ is bounded and $M \in C_{\mathrm{c}}^{2}\left(\mathbb{R}, X^{*}\right)$. 
Now let $Y$ be arbitrary. Let $f \in C^{\alpha}(\mathbb{R}, X)$ be bounded and $M \in$ $C_{\mathrm{c}}^{2}(\mathbb{R}, \mathcal{L}(X, Y))$. For each $y \in Y^{*}$, we can apply (2.8) with $M$ replaced by $y^{*} \circ M$. This gives

$$
\left\|y^{*} \circ(\mathcal{F} M * f)\right\|_{\alpha}+\left\|y^{*} \circ(\mathcal{F} M * f)\right\|_{\infty} \leq c\left\|y^{*}\right\|\|M\| \tilde{\mathcal{M}}_{1}\left(\|f\|_{\alpha}+\|f\|_{\infty}\right) .
$$

By the Hahn-Banach theorem,

$$
\|\mathcal{F} M * f\|_{\alpha}=\sup _{\left\|y^{*}\right\| \leq 1}\left\|y^{*} \circ(\mathcal{F} M * f)\right\|_{\alpha} \leq c\|M\| \tilde{\mathcal{M}}_{1}\left(\|f\|_{\alpha}+\|f\|_{\infty}\right) .
$$

Next, take $\lambda>0$ and let $f_{\lambda}(t)=f(\lambda t)$ and $M_{\lambda^{-1}}(t)=M\left(\lambda^{-1} t\right)$. Then

$$
\begin{aligned}
\left\|f_{\lambda}\right\|_{\alpha} & =\lambda^{\alpha}\|f\|_{\alpha} \\
\left\|M_{\lambda^{-1}}\right\|_{\mathcal{M}_{1}} & \leq\left\|M_{\lambda^{-1}}\right\|_{\mathcal{M}_{1}}+\left\|\left(M_{\lambda^{-1}}\right)^{\prime}\right\|_{\infty}=\|M\|_{\mathcal{M}_{1}}+\lambda^{-1}\left\|M^{\prime}\right\|_{\infty} \\
\left(\mathcal{F} M_{\lambda^{-1}}\right)(s) & =\lambda(\mathcal{F} M)(\lambda s) \\
(\mathcal{F} M * f)(t) & =\left(\mathcal{F} M_{\lambda^{-1}} * f_{\lambda}\right)\left(\lambda^{-1} t\right) .
\end{aligned}
$$

It follows from these relations and (2.9) that

$$
\begin{aligned}
\|\mathcal{F} M * f\|_{\alpha} & =\lambda^{-\alpha}\left\|\mathcal{F} M_{\lambda^{-1}} * f_{\lambda}\right\|_{\alpha} \\
& \leq c\left\|M_{\lambda^{-1}}\right\|_{\mathcal{M}_{1}} \lambda^{-\alpha}\left(\left\|f_{\lambda}\right\|_{\alpha}+\left\|f_{\lambda}\right\|_{\infty}\right) \\
& \leq c\left(\|M\|_{\mathcal{M}_{1}}+\lambda^{-1}\left\|M^{\prime}\right\|_{\infty}\right)\left(\|f\|_{\alpha}+\lambda^{-\alpha}\|f\|_{\infty}\right) .
\end{aligned}
$$

Letting $\lambda \rightarrow \infty$ gives

$$
\|\mathcal{F} M * f\|_{\alpha} \leq c\|M\|_{\mathcal{M}_{1}}\|f\|_{\alpha}
$$

when $f$ is bounded.

Now suppose that $f \in C^{\alpha}(\mathbb{R}, X)$ is arbitrary. Let

$$
f_{n}(t)= \begin{cases}f(t) & \text { if }\|f(t)\| \leq n, \\ n f(t) /\|f(t)\| & \text { otherwise. }\end{cases}
$$

Then $f_{n}$ is bounded, $f_{n} \in C^{\alpha}(\mathbb{R}, X),\left\|f_{n}\right\|_{\alpha} \leq 2\|f\|_{\alpha},\left\|f_{n}(t)\right\| \leq\|f(t)\|$ and $f(t)=\lim _{n \rightarrow \infty} f_{n}(t)$. By the dominated convergence theorem,

$$
(\mathcal{F} M * f)(t)=\lim _{n \rightarrow \infty}\left(\mathcal{F} M * f_{n}\right)(t) .
$$

Applying (2.10) to $f_{n}$ gives

$$
\|\mathcal{F} M * f\|_{\alpha} \leq \limsup _{n \rightarrow \infty}\left\|\mathcal{F} M * f_{n}\right\|_{\alpha} \leq 2 c\|M\|_{\mathcal{M}_{1}}\|f\|_{\alpha}
$$

For simplicity, we have given the results above (and we will give the multiplier theorems in Sections 3 and 5) for functions on $\mathbb{R}$. There are straightforward generalizations to functions on $\mathbb{R}^{N}$ which we state here. Let $k \in \mathbb{N}$ and $M \in C^{k}\left(\mathbb{R}^{N} \backslash\{0\}, \mathcal{L}(X, Y)\right)$. The Mikhlin norm of order $k$ is defined by

$$
\|M\|_{\mathcal{M}_{k}}=\sum_{|\beta| \leq k} \sup _{t \in \mathbb{R}^{N} \backslash\{0\}}|t|^{|\beta|}\left\|D^{\beta} M(t)\right\|,
$$


where the sum is taken over all multi-indices $\beta=\left(\beta_{1}, \ldots, \beta_{N}\right) \in(\mathbb{N} \cup\{0\})^{N}$ with $|\beta|:=\beta_{1}+\ldots+\beta_{N} \leq k$.

Proposition 2.9. Let $X$ be a Banach space of Fourier type $p \in[1,2]$ and $Y$ be arbitrary. Let $k$ be an integer such that $k>N / p$ (for example, $k=N+1$ for arbitrary $X)$. For each $\alpha \in(0,1)$, there is a constant $c_{\alpha, N, X}$ (depending on $\alpha, N$ and $p$ ) such that

$$
\|\mathcal{F} M * f\|_{\alpha} \leq c_{\alpha, N, X}\|M\|_{\mathcal{M}_{k}}\|f\|_{\alpha}
$$

for all $f \in C^{\alpha}\left(\mathbb{R}^{N}, X\right)$ and all $M \in C_{\mathrm{c}}^{N+1}\left(\mathbb{R}^{N}, \mathcal{L}(X, Y)\right)$.

The proof is very similar to that of Proposition 2.1 (for the case when $p=1, k=N+1$ and $X$ is arbitrary) or Proposition 2.8 (for general $p$ ).

3. Periodic multipliers. Let $X, Y$ be Banach spaces, $0<\alpha<1$ and $\tau>0$. By $C_{\tau}(\mathbb{R}, X)$ we denote the space of all $\tau$-periodic continuous functions $f: \mathbb{R} \rightarrow X$. We let $C_{\tau}^{\alpha}(\mathbb{R}, X)=C^{\alpha}(\mathbb{R}, X) \cap C_{\tau}(\mathbb{R}, X)$. Observe that $C_{\tau}^{\alpha}(\mathbb{R}, X)$ is a Banach space for the norm

$$
\|f\|_{C_{\tau}^{\alpha}}:=\|f\|_{\alpha}+\left\|\frac{1}{\tau} \int_{0}^{\tau} f(t) d t\right\|
$$

and that convergence in this norm implies uniform convergence. Our aim is to prove a multiplier theorem for $C_{\tau}^{\alpha}(\mathbb{R}, X)$. Without loss of generality, we shall take $\tau=2 \pi$.

For $x \in X, k \in \mathbb{Z}$ we let $\left(e_{k} \otimes x\right)(t)=e^{i k t} x(t \in \mathbb{R})$. For $k \neq 0$,

$$
\left\|e_{k} \otimes x\right\|_{C_{2 \pi}^{\alpha}}=\left\|e_{k} \otimes x\right\|_{\alpha}=\gamma_{\alpha}|k|^{\alpha}\|x\|,
$$

where $\gamma_{\alpha}=\left\|e_{1}\right\|_{\alpha}=2 \sup _{t>0} t^{-\alpha} \sin (t / 2)$. A trigonometric polynomial is a function $p$ of the form $p=\sum_{k \in \mathbb{Z}} e_{k} \otimes x_{k}$ where $x_{k} \in X$ and $x_{k}=0$ for all but finitely many $k \in \mathbb{Z}$.

Let $f \in C_{2 \pi}(\mathbb{R}, X)$. We denote by

$$
\widehat{f}(k)=\frac{1}{2 \pi} \int_{0}^{2 \pi} f(t) e^{-i k t} d t
$$

the $k$ th Fourier coefficient of $f(k \in \mathbb{Z})$. By Fejér's theorem

$$
\sigma_{n}(f):=\frac{1}{n+1} \sum_{l=0}^{n} \sum_{k=-l}^{l} e_{k} \otimes \widehat{f}(k)=\sum_{k=-n}^{n}\left(1-\frac{|k|}{n+1}\right) e_{k} \otimes \widehat{f}(k)
$$

converges uniformly to $f$ as $n \rightarrow \infty$ (see [ABHN, Theorem 4.2.19]). Moreover, $\sigma_{n}$ is given as convolution by the Fejér kernel

$$
F_{n}(t)= \begin{cases}\frac{1}{n+1}\left(\frac{\sin ((n+1) t / 2)}{\sin (t / 2)}\right)^{2} & (t \notin 2 \pi \mathbb{Z}), \\ n+1 & (t \in 2 \pi \mathbb{Z})\end{cases}
$$


i.e.,

$$
\sigma_{n}(f)(t)=\frac{1}{2 \pi} \int_{-\pi}^{\pi} f(t-s) F_{n}(s) d s .
$$

The uniqueness theorem asserts that a function $f \in C_{2 \pi}(\mathbb{R}, X)$ is 0 whenever $\widehat{f}(k)=0$ for all $k \in \mathbb{Z}$. This is a direct consequence of Fejér's theorem.

Definition 3.1. A sequence $\left(M_{k}\right)_{k \in \mathbb{Z}} \subset \mathcal{L}(X, Y)$ is called a $C_{2 \pi}^{\alpha}$-multiplier if for each $f \in C_{2 \pi}^{\alpha}(\mathbb{R}, X)$ there exists a $u \in C_{2 \pi}^{\alpha}(\mathbb{R}, Y)$ such that

$$
\widehat{u}(k)=M_{k} \widehat{f}(k) \quad \text { for all } k \in \mathbb{Z} .
$$

It follows from the uniqueness theorem that $u$ is uniquely determined by $f$. If $\left(M_{k}\right)_{k \in \mathbb{Z}}$ is a $C_{2 \pi}^{\alpha}$-multiplier then the uniqueness theorem and the closed graph theorem show that the mapping

$$
M: f \mapsto u: C_{2 \pi}^{\alpha}(\mathbb{R}, X) \rightarrow C_{2 \pi}^{\alpha}(\mathbb{R}, Y)
$$

is linear and continuous. We call it the operator associated with the $C_{2 \pi^{-}}^{\alpha}$ multiplier $\left(M_{k}\right)_{k \in \mathbb{Z}}$. Clearly,

$$
M\left(\sum_{k \in \mathbb{Z}} e_{k} \otimes x_{k}\right)=\sum_{k \in \mathbb{Z}} e_{k} \otimes M_{k} x_{k}
$$

for each trigonometric polynomial. Note however that the space of all trigonometric polynomials is not dense in $C_{2 \pi}^{\alpha}(\mathbb{R}, X)$ (for example, the function $|\sin t|^{\alpha}$ is not in the closure of the trigonometric polynomials).

Lemma 3.2. If $\left(M_{k}\right)_{k \in \mathbb{Z}} \subset \mathcal{L}(X, Y)$ is a $C_{2 \pi}^{\alpha}$-multiplier, then

$$
\sup _{k \in \mathbb{Z}}\left\|M_{k}\right\|<\infty \text {. }
$$

Proof. Let $x \in X, x \neq 0, k \in \mathbb{Z} \backslash\{0\}$. Then $M\left(e_{k} \otimes x\right)=e_{k} \otimes M_{k} x$. Hence

$$
\begin{aligned}
\gamma_{\alpha}|k|^{\alpha}\left\|M_{k} x\right\| & =\left\|e_{k} \otimes M_{k} x\right\|_{C_{2 \pi}^{\alpha}}=\left\|M\left(e_{k} \otimes x\right)\right\|_{C_{2 \pi}^{\alpha}} \\
& \leq\|M\|\left\|e_{k} \otimes x\right\|_{C_{2 \pi}^{\alpha}}^{\alpha}=\|M\| \gamma_{\alpha}|k|^{\alpha}\|x\| .
\end{aligned}
$$

Since $M_{0}$ is bounded, the claim follows.

The following definition is the discrete analogue of the Mikhlin norm defined in Section 2.

Definition 3.3. A sequence $\left(M_{k}\right)_{k \in \mathbb{Z}} \subset \mathcal{L}(X, Y)$ satisfies a Marcinkiewicz estimate of order 1 if it is bounded and $\sup _{k \in \mathbb{Z}}\left\|k\left(M_{k+1}-M_{k}\right)\right\|<\infty$. If in addition $\sup _{k \in \mathbb{Z}} k^{2}\left\|M_{k+1}-2 M_{k}+M_{k-1}\right\|<\infty$ then we say that $\left(M_{k}\right)_{k \in \mathbb{Z}}$ satisfies a Marcinkiewicz estimate of order 2 . We let

$$
\begin{aligned}
& \left\|\left(M_{k}\right)_{k \in \mathbb{Z}}\right\|_{\mathcal{M}(\mathbb{Z})} \\
& \quad=\sup _{k}\left\|M_{k}\right\|+\sup _{k}\left\|k\left(M_{k+1}-M_{k}\right)\right\|+\sup _{k}\left\|k^{2}\left(M_{k+1}-2 M_{k}+M_{k-1}\right)\right\|
\end{aligned}
$$


and denote by $\mathcal{M}(\mathbb{Z})$ the space of all sequences such that $\left\|\left(M_{k}\right)_{k \in \mathbb{Z}}\right\|_{\mathcal{M}(\mathbb{Z})}$ $<\infty$.

Now we formulate the periodic multiplier theorem.

THEOREM 3.4. If $\left(M_{k}\right)_{k \in \mathbb{Z}} \subset \mathcal{L}(X, Y)$ satisfies a Marcinkiewicz estimate of order 2 , then $\left(M_{k}\right)_{k \in \mathbb{Z}}$ is a $C_{2 \pi}^{\alpha}$-multiplier.

For the proof we need the following extension lemma.

LEMMA 3.5. There exists a constant $c_{\mathcal{M}}>0$ such that for each $\left(M_{k}\right)_{k \in \mathbb{Z}}$ $\in \mathcal{M}(\mathbb{Z})$ there exists $\bar{M} \in C^{\infty}(\mathbb{R}, \mathcal{L}(X, Y))$ such that

$$
\bar{M}(k)=M_{k}(k \in \mathbb{Z}) \quad \text { and } \quad\|\bar{M}\|_{\mathcal{M}} \leq c_{\mathcal{M}}\left\|\left(M_{k}\right)_{k \in \mathbb{Z}}\right\|_{\mathcal{M}(\mathbb{Z})} .
$$

Moreover, if $M_{k}=0$ for all but finitely many $k$, one may choose $\bar{M}$ of compact support.

Proof. Let $\Phi:[0,1] \rightarrow \mathbb{R}$ be a $C^{\infty}$-function such that $\Phi(t)=0$ whenever $0 \leq t \leq 1 / 4$ and $\Phi(t)=t-1$ whenever $3 / 4 \leq t \leq 1$. Define

$$
\bar{M}(k+t)=(1-t) M_{k}+t M_{k+1}+\left(M_{k+2}-2 M_{k+1}+M_{k}\right) \Phi(t) \quad(0 \leq t<1) .
$$

Then

$$
\begin{aligned}
\lim _{t \uparrow 1} \bar{M}(k+t) & =M_{k+1}=\lim _{t \downarrow 0} M(k+1+t), \\
\lim _{t \uparrow 1} \frac{\bar{M}(k+1)-\bar{M}(k+t)}{1-t} & =M_{k+2}-M_{k+1}=\lim _{t \downarrow 0} \frac{M(k+1+t)-M(k+1)}{t}, \\
\bar{M}^{\prime \prime}(k+t) & =0 \quad(|t| \leq 1 / 4) .
\end{aligned}
$$

So $\bar{M} \in C^{\infty}(\mathbb{R}, \mathcal{L}(X, Y))$. Furthermore,

$$
\begin{aligned}
\|\bar{M}(k+t)\| & \leq \sup _{r}\left\|M_{r}\right\|_{\infty}+\|\Phi\|_{\infty}\left\|M_{k+2}-2 M_{k+1}+M_{k}\right\| \\
& \leq\left(1+4\|\Phi\|_{\infty}\right) \sup _{r}\left\|M_{r}\right\| .
\end{aligned}
$$

Moreover,

$$
\begin{aligned}
\|(k+ & t) \bar{M}^{\prime}(k+t) \| \\
\leq & (|k|+1)\left\|M_{k+1}-M_{k}\right\|+|k+t|\left\|\Phi^{\prime}\right\|_{\infty}\left\|M_{k+2}-2 M_{k+1}+M_{k}\right\| \\
\leq & (|k|+1)\left\|M_{k+1}-M_{k}\right\| \\
& \quad+\left\|\Phi^{\prime}\right\|_{\infty}\left((|k|+1)\left\|M_{k+1}-M_{k}\right\|+(|k+1|+1)\left\|M_{k+2}-M_{k+1}\right\|\right) \\
\leq & \left(1+2\left\|\Phi^{\prime}\right\|_{\infty}\right) \sup _{r}|r|\left\|M_{r+1}-M_{r}\right\|+\left(2+4\left\|\Phi^{\prime}\right\|_{\infty}\right) \sup _{r}\left\|M_{r}\right\| .
\end{aligned}
$$

Finally,

$$
\begin{aligned}
\left\|(k+t)^{2} \bar{M}^{\prime \prime}(k+t)\right\| & =(k+t)^{2}\left\|M_{k+2}-2 M_{k+1}+M_{k}\right\|\left\|\Phi^{\prime \prime}\right\|_{\infty} \\
& \leq\left((k+1)^{2}+2|k|-1\right)\left\|M_{k+2}-2 M_{k+1}+M_{k}\right\|\left\|\Phi^{\prime \prime}\right\|_{\infty}
\end{aligned}
$$




$$
\begin{aligned}
\leq & \left\{(k+1)^{2}\left\|M_{k+2}-2 M_{k+1}+M_{k}\right\|+2|k|\left\|M_{k+1}-M_{k}\right\|\right. \\
& \left.+2|k+1|\left\|M_{k+2}-M_{k+1}\right\|+\left\|M_{k+2}\right\|+\left\|M_{k+1}\right\|\right\}\left\|\Phi^{\prime \prime}\right\|_{\infty} \\
\leq & 4\left\|\left(M_{r}\right)_{r \in \mathbb{Z}}\right\|_{\mathcal{M}(\mathbb{Z})}\left\|\Phi^{\prime \prime}\right\|_{\infty} .
\end{aligned}
$$

Proof of Theorem 3.4. (a) Let $f=\sum_{|k| \leq N} e_{k} \otimes \widehat{f}(k)$ be a trigonometric polynomial. We show that

$$
\left\|\sum_{|k| \leq N} e_{k} \otimes M_{k} \widehat{f}(k)\right\|_{\alpha} \leq \bar{c}\left\|\sum_{|k| \leq N} e_{k} \otimes \widehat{f}(k)\right\|_{\alpha}
$$

where

$$
\bar{c}=\frac{c_{\alpha} c_{\mathcal{M}} \kappa^{2}}{2 \pi}\left\|\left(M_{k}\right)_{k \in \mathbb{Z}}\right\|_{\mathcal{M}(\mathbb{Z})}
$$

By Lemma 3.5 and Corollary 2.3, there exists $\widetilde{M} \in C_{\mathrm{c}}^{\infty}(\mathbb{R}, \mathcal{L}(X, Y))$ such that $\widetilde{M}(k)=M_{-k}(|k| \leq N)$ and $\|\widetilde{M}\|_{\mathcal{M}} \leq c_{\mathcal{M}} \kappa^{2}\left\|\left(M_{k}\right)_{k \in \mathbb{Z}}\right\|_{\mathcal{M}(\mathbb{Z})}$. One has

$$
\begin{aligned}
\sum_{|k| \leq N} e^{i k t} M_{k} \widehat{f}(k) & =\sum_{|k| \leq N} e^{i k t}\left(\mathcal{F}^{-1} \mathcal{F} \widetilde{M}\right)(-k) \widehat{f}(k) \\
& =\frac{1}{2 \pi} \sum_{|k| \leq N} \int_{\mathbb{R}}(\mathcal{F} \widetilde{M})(s) e^{-i k s} d s \widehat{f}(k) e^{i k t} \\
& =\frac{1}{2 \pi}(\mathcal{F} \widetilde{M} * f)(t) .
\end{aligned}
$$

The basic estimate (2.5) of Proposition 2.1 gives

$$
\|\mathcal{F} \bar{M} * f\|_{\alpha} \leq c_{\alpha}\|\bar{M}\|_{\mathcal{M}}\|f\|_{\alpha}
$$

Now the claim follows.

(b) Assume that $Y=\mathbb{C}$. Let $f \in C_{2 \pi}^{\alpha}(\mathbb{R}, X)$. We show that there exists $u \in C_{2 \pi}^{\alpha}(\mathbb{R}, \mathbb{C})$ such that $\|u\|_{\alpha} \leq \bar{c}\|f\|_{\alpha}$ and $\widehat{u}(k)=M_{k} \widehat{f}(k)(k \in \mathbb{Z})$. Let $f_{n}=\sigma_{n}(f)$. Since $(2 \pi)^{-1} \int_{-\pi}^{\pi} F_{n}(s) d s=1$, it follows from (3.3) that $\left\|f_{n}\right\|_{\alpha} \leq\|f\|_{\alpha}(n \in \mathbb{N})$. Let $u_{n}=\sum_{|k| \leq n} e_{k} \otimes M_{k} \widehat{f}_{n}(k)$. Then $\left\|u_{n}\right\|_{\alpha} \leq$ $\bar{c}\left\|f_{n}\right\|_{\alpha} \leq \bar{c}\|f\|_{\alpha}$ by (a). Moreover, $\widehat{u}_{n}(0)=M_{0} \widehat{f}(0)$. This implies that $\left(u_{n}\right)$ is equicontinuous and uniformly bounded. It follows from Ascoli's theorem that every subsequence of $\left(u_{n}\right)$ has a subsequence which converges uniformly to a function $u$. Then $\widehat{u}(k)=M_{k} \widehat{f}(k)$ for all $k \in \mathbb{Z}$. This shows that $u$ is independent of the subsequences. Hence $\lim _{n \rightarrow \infty} u_{n}=u$ uniformly, where $u \in C_{2 \pi}^{\alpha}(\mathbb{R}, \mathbb{C})$ and $\|u\|_{\alpha} \leq \lim \sup _{n \rightarrow \infty}\left\|u_{n}\right\|_{\alpha} \leq \bar{c}\|f\|_{\alpha}$.

(c) Let $Y$ be arbitrary. Let $y^{*} \in Y^{*}$. Then by (b) there exists a unique $u_{y^{*}} \in C_{2 \pi}^{\alpha}(\mathbb{R}, \mathbb{C})$ such that $\widehat{u}_{y^{*}}(k)=\left\langle y^{*}, M_{k} \widehat{f}(k)\right\rangle(k \in \mathbb{Z})$. Moreover, $\left\|u_{y^{*}}\right\|_{\alpha} \leq \bar{c}\left\|y^{*}\right\|\|f\|_{\alpha}$. Define $u(t) \in Y^{* *}$ by $\left\langle u(t), y^{*}\right\rangle=u_{y^{*}}(t)$. Then $u \in$ $C_{2 \pi}^{\alpha}\left(\mathbb{R}, Y^{* *}\right)$ and $\left\langle\widehat{u}(k), y^{*}\right\rangle=\left\langle y^{*}, M_{k} \widehat{f}(k)\right\rangle$. Thus, $\widehat{u}(k)=M_{k} \widehat{f}(k) \in Y$ for all $k \in \mathbb{Z}$. Consider the quotient map $q: Y^{* *} \rightarrow Y^{* *} / Y$ (where we consider 
$Y$ as a subspace of $Y^{* *}$ via evaluation). Then $q \circ u \in C_{2 \pi}\left(\mathbb{R}, Y^{* *} / Y\right)$ and $\widehat{q \circ u}(k)=0$ for all $k \in \mathbb{Z}$. Hence $q \circ u(t)=0$ for all $t \in \mathbb{R}$ by the uniqueness theorem. We have shown that $u(t) \in Y$ for all $t \in \mathbb{R}$. Finally, for $t \neq s$ we have

$$
\frac{\|u(t)-u(s)\|}{|t-s|^{\alpha}}=\sup _{\left\|y^{*}\right\| \leq 1} \frac{\left|u_{y^{*}}(t)-u_{y^{*}}(s)\right|}{|t-s|^{\alpha}} \leq \bar{c}\|f\|_{\alpha} .
$$

This completes the proof.

REMARK 3.6. A Marcinkiewicz estimate of order 1 suffices in order that Theorem 3.4 holds true when $X$ is $B$-convex. The proof is the same as above, except that Proposition 2.8 (or the result of Girardi and Weis [GW, Corollary 2.10]) is used instead of Proposition 2.1. We do not know whether the same result is true for general Banach spaces.

EXAMPLE 3.7 (Hilbert transform and Riesz projection). Let $X$ be an arbitrary Banach space and $0<\alpha<1$. Let $M_{k}=I$ for $k \geq 0$ and $M_{k}=0$ for $k<0$. Then $\left(M_{k}\right)_{k \in \mathbb{Z}}$ is a $C_{2 \pi}^{\alpha}$-multiplier by Theorem 3.4. The associated operator is called the Riesz projection. Similarly, letting $M_{k}=(-i \operatorname{sign} k) I$ defines a $C_{2 \pi}^{\alpha}$-multiplier. The associated operator on $C_{2 \pi}^{\alpha}(\mathbb{R}, X)$ is called the Hilbert transform.

4. Periodic boundary conditions. In this section we apply the multiplier theorem (Theorem 3.4) to differential equations with periodic boundary conditions. It is remarkable that in this context (i.e., applying the multiplier theorem to resolvents) one obtains a complete characterization of well-posedness. At the end of the section we consider Dirichlet boundary conditions and also the second order problem with diverse boundary conditions.

Let $A$ be a closed operator on a Banach space $X$, and let $\tau>0$. For $f \in C([0, \tau], X)$ we consider the problem

$\left(\mathrm{P}_{\mathrm{per}}(f)\right)$

$$
\left\{\begin{array}{l}
u^{\prime}(t)=A u(t)+f(t) \quad(t \in[0, \tau]), \\
u(0)=u(\tau)
\end{array}\right.
$$

A classical solution of $\left(\mathrm{P}_{\mathrm{per}}(f)\right)$ is a function $u \in C^{1}([0, \tau], X)$ such that $u(t) \in D(A)$ for all $t \in[0, \tau]$ and $\left(\mathrm{P}_{\text {per }}(f)\right)$ is satisfied. A mild solution is a function $u \in C([0, \tau], X)$ such that $u(0)=u(\tau)$ and

$$
\int_{0}^{t} u(s) d s \in D(A) \quad \text { and } \quad u(t)=u(0)+A \int_{0}^{t} u(s) d s+\int_{0}^{t} f(s) d s
$$

for all $t \in[0, \tau]$. Since $A$ is closed, each classical solution is a mild solution. Moreover, a mild solution $u$ is a classical solution if and only if $u \in C^{1}([0, \tau], X)($ cf. [ABHN, Section 3.1]). 
Denote by $C_{\text {per }}([0, \tau], X)$ the space of all continuous functions $f:[0, \tau] \rightarrow$ $X$ such that $f(0)=f(\tau)$. Let $0<\alpha<1$. For $f:[0, \tau] \rightarrow X$, let

$$
\|f\|_{\alpha}=\sup \left\{\frac{\|f(t)-f(s)\|}{|t-s|^{\alpha}}: s, t \in[0, \tau], s \neq t\right\} .
$$

Define

$$
\begin{aligned}
C_{\text {per }}^{\alpha}([0, \tau], X) & =\left\{f \in C_{\text {per }}([0, \tau], X):\|f\|_{\alpha}<\infty\right\}, \\
C_{\text {per }}^{1+\alpha}([0, \tau], X) & =\left\{f \in C^{1}([0, \tau], X): f(0)=f(\tau), f^{\prime} \in C_{\text {per }}^{\alpha}([0, \tau], X)\right\} .
\end{aligned}
$$

It is easy to see that the $\tau$-periodic extension $\tilde{f}$ of a function $f \in C_{\text {per }}^{\alpha}([0, \tau], X)$ to $\mathbb{R}$ satisfies

$$
\sup \left\{\frac{\|\widetilde{f}(t)-\widetilde{f}(s)\|}{|t-s|^{\alpha}}: s, t \in \mathbb{R}, s \neq t\right\} \leq 2\|f\|_{\alpha} .
$$

Thus we may identify $C_{\text {per }}^{\alpha}([0, \tau], X)$ with the space $C_{\tau}^{\alpha}(\mathbb{R}, X)$ introduced in Section 3.

Definition 4.1. We say that the problem $\left(\mathrm{P}_{\text {per }}\right)$ is $C^{\alpha}$-well-posed if for every $f \in C_{\text {per }}^{\alpha}([0, \tau], X)$ there exists a unique classical solution $u \in$ $C_{\text {per }}^{1+\alpha}([0, \tau], X)$ of $\left(\mathrm{P}_{\text {per }}(f)\right)$.

Based on the multiplier theorem (Theorem 3.4) it is now possible to characterize $C^{\alpha}$-well-posedness by properties of the resolvent.

Theorem 4.2. Let $A$ be a closed operator and let $\tau>0,0<\alpha<1$. The following assertions are equivalent:

(i) the problem $\left(\mathrm{P}_{\text {per }}\right)$ is $C^{\alpha}$-well-posed;

(ii) $(2 \pi i / \tau) \mathbb{Z} \subset \varrho(A)$ and $\sup _{k \in \mathbb{Z}}\|k R(2 \pi i k / \tau, A)\|<\infty$.

Replacing $A$ by $\frac{\tau}{2 \pi} A$ we can assume that $\tau=2 \pi$, which we will do throughout what follows. We need the following lemma whose proof is similar to [AB1, Lemma 3.1].

Lemma 4.3. Let $v, g \in C_{\mathrm{per}}([0,2 \pi], X)$. The following are equivalent:

(i) $\widehat{v}(k) \in D(A)$ and $A \widehat{v}(k)=\widehat{g}(k)$ for all $k \in \mathbb{Z}$;

(ii) $v(t) \in D(A)$ and $A v(t)=g(t)$ for all $t \in[0,2 \pi]$.

Now mild solutions can be described as follows.

Proposition 4.4. Let $u, f \in C_{\text {per }}([0,2 \pi], X)$. The following assertions are equivlent:

(i) $u$ is a mild solution of $\left(\mathrm{P}_{\mathrm{per}}(f)\right)$;

(ii) $\widehat{u}(k) \in D(A)$ and $(i k-A) \widehat{u}(k)=\widehat{f}(k)$ for all $k \in \mathbb{Z}$.

Proof. First case: Assume that $\widehat{u}(0)=\widehat{f}(0)=0$. Then $v(t):=\int_{0}^{t} u(s) d s$ and $g(t):=u(t)-u(0)-\int_{0}^{t} f(s) d s$ define functions $v, g \in C_{\text {per }}([0,2 \pi], X)$. 
(a) Assume that $u$ is a mild solution of $\left(\mathrm{P}_{\text {per }}(f)\right)$. By Lemma 4.3, $\widehat{u}(k)=$ $i k \widehat{v}(k) \in D(A)$ and $A \widehat{u}(k)=i k \widehat{g}(k)=i k \widehat{u}(k)-\widehat{f}(k)$ for all $k$. This shows that condition (ii) holds.

(b) Conversely, assume (ii). Consider $v_{1}(t):=v(t)-x, g_{1}(t):=g(t)-y$ where $x=\widehat{v}(0), y=\widehat{g}(0)$. Then $\widehat{v}_{1}(0)=\widehat{g}_{1}(0)=0$ and $\widehat{v}_{1}(k)=\widehat{v}(k)=$ $\widehat{u}(k) / i k$ and $\widehat{g}_{1}(k)=\widehat{u}(k)-\widehat{f(k)} / i k$ for $k \neq 0$. Thus $A \widehat{v}_{1}(k)=\widehat{g}_{1}(k)$ for all $k \in \mathbb{Z}$. It follows from Lemma 4.3 that $v_{1}(t) \in D(A)$ and $A v_{1}(t)=g_{1}(t)$ for all $t \in[0,2 \pi]$. Choosing $t=0$ we conclude that $-x=v_{1}(0) \in D(A)$ and $-A x=-y=g_{1}(0)$. Hence $\int_{0}^{t} u(s) d s \in D(A)$ and $A \int_{0}^{t} u(s) d s=g(t)$ for all $t \in[0,2 \pi]$; that is, $u$ is a mild solution of $\left(\mathrm{P}_{\text {per }}(f)\right)$.

Second case: $u, f$ are arbitrary. Consider $u_{1}(t):=u(t)-\widehat{u}(0), f_{1}(t):=$ $f(t)-\widehat{f}(0)$. Then $\widehat{u}_{1}(k)=\widehat{u}(k), \widehat{f}_{1}(k)=\widehat{f}(k)$ for all $k \in \mathbb{Z} \backslash\{0\}$ and $\widehat{u}_{1}(0)=\widehat{f}_{1}(0)=0$.

(a) Assume that $u$ is a mild solution of $\left(\mathrm{P}_{\mathrm{per}}(f)\right)$. Letting $t=2 \pi$ in (4.1), one sees that $\widehat{u}(0) \in D(A)$ and $-A \widehat{u}(0)=\widehat{f}(0)$. Thus $u_{1}$ is a mild solution of $\left(\mathrm{P}_{\text {per }}\left(f_{1}\right)\right)$. It follows from the first case that $\widehat{u}(k)=\widehat{u}_{1}(k) \in D(A)$ and $(i k-A) \widehat{u}(k)=(i k-A) \widehat{u}_{1}(k)=\widehat{f}_{1}(k)=\widehat{f}(k)$ for all $k \in \mathbb{Z} \backslash\{0\}$.

(b) Conversely, assume (ii). Then $\widehat{u}_{1}(k) \in D(A)$ and $(i k-A) \widehat{u}_{1}(k)=$ $\widehat{f}_{1}(k)$ for all $k \in \mathbb{Z}$. It follows from the first case that $u_{1}$ is a mild solution of $\left(\mathrm{P}_{\text {per }}\left(f_{1}\right)\right)$; that is, $\int_{0}^{t} u(s) d s-t \widehat{u}(0) \in D(A)$ and $A\left(\int_{0}^{t} u(s) d s-t \widehat{u}(0)\right)=$ $u_{1}(t)-u_{1}(0)-\int_{0}^{t} f_{1}(s) d s=u(t)-u(0)-\int_{0}^{t} f(s) d s+t \widehat{f}(0)$. Since by the assumption $\widehat{u}(0) \in D(A)$ and $-A \widehat{u}(0)=\widehat{f}(0)$, this implies that $u$ is a mild solution.

Proof of Theorem 4.2. We assume that $\tau=2 \pi$. Assume that $i \mathbb{Z} \subset \varrho(A)$ and $\sup _{k \in \mathbb{Z}}\|k R(i k, A)\|<\infty$. Let $M_{k}=k R(i k, A)(k \in \mathbb{Z})$. We show that $\left(M_{k}\right)_{k \in \mathbb{Z}}$ satisfies the Marcinkiewicz condition of order 2 in $\mathcal{L}(X)$.

Let $B=-i A$ and $R(k)=R(k, B)=i R(i k, A)$. Then the resolvent identity gives

$$
\begin{aligned}
i\left(M_{k+1}-M_{k}\right) & =(k+1) R(k+1)-k R(k) \\
& =R(k+1)+k(R(k+1)-R(k)) \\
& =R(k+1)(I-k R(k)) .
\end{aligned}
$$

It follows that $\sup _{k \in \mathbb{Z}}\left\|k\left(M_{k+1}-M_{k}\right)\right\|<\infty$. The order-2-condition is obtained by

$$
\begin{aligned}
i\left(M_{k+1}\right. & \left.-2 M_{k}+M_{k-1}\right) \\
& =(k+1) R(k+1)-k R(k)+(k-1) R(k-1)-k R(k) \\
& =R(k+1)-R(k-1)+k(R(k+1)-R(k)+R(k-1)-R(k))
\end{aligned}
$$




$$
\begin{aligned}
& =R(k+1)-R(k-1)-k R(k+1) R(k)+k R(k-1) R(k) \\
& =(R(k+1)-R(k-1))(I-k R(k)) \\
& =2 R(k+1) R(k-1)(I-k R(k)) .
\end{aligned}
$$

This shows that $\sup _{k \in \mathbb{Z}}\left\|k^{2}\left(M_{k+1}-2 M_{k}+M_{k-1}\right)\right\|<\infty$.

Let $f \in C_{2 \pi}^{\alpha}(\mathbb{R}, X)$. Since $A$ is invertible and $A R(i k, A)=i k R(i k, A)-I$ it follows from Theorem 3.4 that $(R(i k, A))_{k \in \mathbb{Z}} \subset \mathcal{L}(X, D(A))$ is a $C_{2 \pi^{-}}^{\alpha}$ multiplier, where $D(A)$ is considered as a Banach space in the graph norm. Hence there exists $u \in C_{2 \pi}^{\alpha}(\mathbb{R}, D(A))$ such that $R(i k, A) \widehat{f}(k)=\widehat{u}(k)$ for all $k \in \mathbb{Z}$. It follows from Proposition 4.4 that $u$ is a mild solution of $\left(\mathrm{P}_{\mathrm{per}}(f)\right)$. Since $(i k R(i k, A))_{k \in \mathbb{Z}}$ is a $C_{2 \pi}^{\alpha}$-multiplier in $\mathcal{L}(X)$, there exists $v \in C_{2 \pi}^{\alpha}(\mathbb{R}, X)$ such that $\widehat{v}(k)=i k R(i k, A) \widehat{f}(k)$ for all $k \in \mathbb{Z}$. Hence $\widehat{v}(k)=i k \widehat{u}(k)$ for all $k \in \mathbb{Z}$. It follows from the proof of [AB1, Lemma 2.1] that $u$ is differentiable and $u^{\prime}=v$. Thus $u$ is a classical solution of $\left(\mathrm{P}_{\text {per }}(f)\right)$. Uniqueness follows from Proposition 4.4.

This finishes the proof of one implication. The other is proved as [AB1, Theorem $2.3(\mathrm{i}) \Rightarrow(\mathrm{ii})]$.

REMARK 4.5. Using the same techniques as in Theorem 4.2 , it is possible to treat a more general situation. Suppose that $i \mathbb{Z} \backslash\{0\} \subset \varrho(A)$ and $\sup _{k \in \mathbb{Z} \backslash\{0\}}\|k R(i k, A)\|<\infty$. Let $f \in C_{\text {per }}^{\alpha}([0,2 \pi], X)$. Then $\left(\mathrm{P}_{\text {per }}(f)\right)$ has a mild or classical solution $u \in C_{\text {per }}([0,2 \pi], X)$ if and only if $\widehat{f}(0)$ belongs to the range of $A$. In that case $\left.u \in C_{\text {per }}^{1+\alpha}([0,2 \pi]), X\right)$ and $u$ is given by

$$
u(t)=x+\int_{0}^{t} v(s) d s,
$$

where $x \in D(A)$ and $A x=-\widehat{f}(0)$, and

$$
\widehat{v}(k)= \begin{cases}i k R(i k, A) \widehat{f} & (k \neq 0), \\ 0 & (k=0) .\end{cases}
$$

The existence of such $v \in C_{\mathrm{per}}^{\alpha}([0,2 \pi], X)$ follows from Theorem 3.4.

Next we apply the multiplier theorem to establish the existence of mild solutions under a weaker growth condition on the resolvent.

TheOREM 4.6. Let $A$ be a closed operator such that $i \mathbb{Z} \subset \varrho(A)$ and

$$
\sup _{k \in \mathbb{Z}}|k|^{2 / 3}\|R(i k, A)\|<\infty .
$$

Then for all $f \in C_{\mathrm{per}}^{\alpha}([0,2 \pi], X)$ there exists a unique mild solution $u \in$ $C_{\text {per }}^{\alpha}([0,2 \pi], X)$ of $\left(\mathrm{P}_{\text {per }}(f)\right)$. 
Proof. Let $M_{k}=R(i k, A)(k \in \mathbb{Z})$. Then

$$
M_{k+1}-M_{k}=-i R(i(k+1), A) R(i k, A)
$$

by the resolvent equation. Moreover,

$$
\begin{aligned}
M_{k+1}-2 M_{k}+M_{k-1} & =\left(M_{k+1}-M_{k}\right)-\left(M_{k}-M_{k-1}\right) \\
& =-i R(i(k+1), A) R(i k, A)+i R(i k, A) R(i(k-1), A) \\
& =i R(i k, A)(R(i(k-1), A)-R(i(k+1), A)) \\
& =i R(i k, A) 2 i R(i(k-1), A) R(i(k+1), A)
\end{aligned}
$$

by the resolvent equation. It follows from these identities that $(R(i k, A))_{k \in \mathbb{Z}}$ satisfies the Marcinkiewicz condition of order 2. Let $f \in C_{\mathrm{per}}^{\alpha}([0,2 \pi], X)$. By Theorem 3.4 there exists $u \in C_{\text {per }}^{\alpha}([0,2 \pi], X)$ such that $\widehat{u}(k)=R(i k, A) \widehat{f}(k)$ $(k \in \mathbb{Z})$. Thus $u$ is the unique mild solution of $\left(\mathrm{P}_{\text {per }}(f)\right)$ by Proposition 4.4.

Remark 4.7. If $X$ is $B$-convex then the growth condition (4.2) in Theorem 4.6 can be weakened to

$$
\sup _{k \in \mathbb{Z}}|k|^{1 / 2}\|R(i k, A)\|<\infty .
$$

This condition implies that $M_{k}:=R(i k, A)$ satisfies a Marcinkiewicz condition of order 1, and then the result follows from Remark 3.6.

Next we consider Dirichlet boundary conditions. Let $\tau>0$. For $f \in$ $C([0, \tau], X)$ and $x \in X$ we consider the problem

$$
\left\{\begin{array}{l}
u^{\prime}(t)=A u(t)+f(t) \quad(t \in[0, \tau]) \\
u(0)=x
\end{array}\right.
$$

Assume that for all $y \in X$, the problem $\left(\mathrm{P}\left(0, f_{y}\right)\right)$ with $f_{y}(t) \equiv y$ has a unique classical solution. Then by [ABHN, Theorem 3.17.1], the operator $A$ generates a $C_{0}$-semigroup $T$ and the solution of $\left(\mathrm{P}\left(0, f_{y}\right)\right)$ is given by $u(t)=\int_{0}^{t} T(s) y d s$. Now assume that $u \in C^{1+\alpha}([0, \tau], X)$. Then $T(\cdot) y=u^{\prime} \in$ $C^{\alpha}([0, \tau], X)$. Consequently, there exists a constant $c_{y} \geq 0$ such that

$$
\frac{\|T(t) y-y\|}{t^{\alpha}} \leq c_{y} \quad(0<t \leq \tau) .
$$

It follows from the closed graph theorem that $\sup _{0<t \leq \tau} t^{-\alpha}\|T(t)-I\|<\infty$. This implies that $A$ is bounded (by [ABHN, Theorem 3.1.10]). Thus, one has maximal regularity for the problem $(\mathrm{P}(0, f))$, i.e., for Dirichlet boundary conditions, only in trivial cases. It is remarkable that periodic boundary conditions are different in this respect, by Theorem 4.2 .

The following result is well known (see the monograph by Lunardi [Lu, Theorem 4.3.1]). We obtain it as a consequence of Theorem 4.2.

COROllary 4.8. Let $A$ be the generator of a holomorphic semigroup. Then for all $x \in X$ and $f \in C^{\alpha}([0, \tau], X)$ there is a unique mild solution $u$ of $(\mathrm{P}(x, f))$. One has $u \in C^{1+\alpha}([\varepsilon, \tau], X)$ for all $\varepsilon \in(0, \tau)$. 
Proof. Let $T$ be the semigroup generated by $A$. Replacing $A$ by $A-\omega$ we may assume that $T$ is exponentially stable. Then condition (ii) of Theorem 4.2 is satisfied. Hence there exists a unique classical solution $v$ of $\left(\mathrm{P}_{\text {per }}(f)\right)$ and $v \in C^{1+\alpha}([0, \tau], X)$. Let $u(t)=v(t)-T(t) v(0)+T(t) x$. Then $u$ is the unique mild solution of $(\mathrm{P}(x, f))$ (see [ABHN, Proposition 3.1.16]). Since $T(\cdot)(x-v(0)) \in C^{\infty}((0, \tau], X)$, one has $u \in C^{1+\alpha}([\varepsilon, \tau], X)$ for all $\varepsilon>0$.

Finally in this section, we consider second order problems. Let $A$ be a closed operator on $X$. The following result is deduced from Theorem 3.4 in much the same way as [AB1, Theorem 6.1] is obtained from the corresponding multiplier theorem on $L^{p}$. We omit the proof. Let $\mathbb{N}_{0}=\mathbb{N} \cup\{0\}$. Let $0<\alpha<1$, and let $C^{2+\alpha}([0,2 \pi], X)$ be the space of all $f \in C^{2}([0,2 \pi], X)$ such that $f^{\prime \prime} \in C^{\alpha}([0,2 \pi], X)$.

THEOREM 4.9. The following assertions are equivalent:

(i) $k^{2} \in \varrho(A)$ for all $k \in \mathbb{N}_{0}$ and $\sup _{k \in \mathbb{N}}\left\|k^{2} R\left(k^{2}, A\right)\right\|<\infty$;

(ii) for all $f \in C_{\mathrm{per}}^{\alpha}([0,2 \pi], X)$ there exists a unique $u \in C^{2+\alpha}([0,2 \pi], X)$ $\cap C^{\alpha}([0,2 \pi], D(A))$ satisfying

$$
\left\{\begin{array}{l}
u^{\prime \prime}(t)+A u(t)=f(t) \quad(t \in[0,2 \pi]) \\
u(0)=u(2 \pi), \quad u^{\prime}(0)=u^{\prime}(2 \pi)
\end{array}\right.
$$

Similarly to [AB1, Theorems 6.3 and 6.4] one may characterize $C^{\alpha}$-wellposedness of the second order problem with Dirichlet and Neumann boundary conditions.

THEOREM 4.10. The following assertions are equivalent:

(i) $k^{2} \in \varrho(A)$ for all $k \in \mathbb{N}$ and $\sup _{k \in \mathbb{N}} k^{2}\left\|R\left(k^{2}, A\right)\right\|<\infty$;

(ii) for all $f \in C_{\mathrm{per}}^{\alpha}([0, \pi], X)$ with $f(0)=0$ there exists a unique function $u \in C^{2+\alpha}([0, \pi], X) \cap C^{\alpha}([0, \pi], D(A))$ satisfying

$$
\left\{\begin{array}{l}
u^{\prime \prime}(t)+A u(t)=f(t) \quad(t \in[0, \pi]), \\
u(0)=u(\pi)=0
\end{array}\right.
$$

THEOREM 4.11. The following assertions are equivalent:

(i) $k^{2} \in \varrho(A)$ for all $k \in \mathbb{N}_{0}$ and $\sup _{k \in \mathbb{N}}\left\|k^{2} R\left(k^{2}, A\right)\right\|<\infty$;

(ii) for all $f \in C^{\alpha}([0, \pi], X)$ there exists a unique $u \in C^{2+\alpha}([0, \pi], X) \cap$ $C^{\alpha}([0, \pi], D(A))$ satisfying

$$
\left\{\begin{array}{l}
u^{\prime \prime}(t)+A u(t)=f(t) \quad(t \in[0, \pi]), \\
u^{\prime}(0)=u^{\prime}(\pi)=0 .
\end{array}\right.
$$

Since Theorem 4.10 can be proved by using an argument similar to the proof of [AB1, Theorem 6.3], we only give the proof for Theorem 4.11. We will use the following lemma. Its simple proof is similar to that of [AB1, Lemma 6.2]. Note that the key point in the proof of [AB1, Lemma 6.2] is 
the boundedness of the Riesz projection on $L^{p}([-\pi, \pi], X)$ when $1<p<\infty$ and $X$ is a UMD-space. In our situation we can use Example 3.7.

Lemma 4.12. Let $\left(M_{k}\right)_{k \in \mathbb{Z}} \subset \mathcal{L}(X)$ be such that $M_{k}=M_{-k}(k \in \mathbb{Z})$. Assume that for each even $f \in C_{\mathrm{per}}^{\alpha}([-\pi, \pi], X)$ there exists $u \in C_{\mathrm{per}}^{\alpha}([-\pi, \pi], X)$ such that $\widehat{u}(k)=M_{k} \widehat{f}(k)(k \in \mathbb{Z})$. Then $\left(M_{k}\right)_{k \in \mathbb{Z}}$ is a $C_{2 \pi}^{\alpha}$-multiplier.

Proof of Theorem 4.11. (ii) $\Rightarrow$ (i). Let $k \in \mathbb{Z}$. If $x \in D(A)$ is such that $\left(-k^{2}+A\right) x=0$, then $u(t)=\cos (k t) x$ satisfies (4.5) with $f=0$. Thus $x=0$ and hence $-k^{2}+A$ is injective. To show surjectivity, take $x \in X$ and let $f(t)=\cos (k t) x$. Let $u$ be the solution of (4.5), and extend $u$ to an even function. Then it is easy to verify that $u$ is twice differentiable on $[-\pi, \pi]$ and $u^{(j)}(-\pi)=u^{(j)}(\pi)$ for $j=0,1,2$. Thus $u \in C_{\text {per }}^{2+\alpha}([-\pi, \pi], X) \cap$ $C_{\mathrm{per}}^{\alpha}([-\pi, \pi], D(A))$ and $u^{\prime \prime}+A u=f$ on $[-\pi, \pi]$. We deduce from Lemma 4.3 that $\left(-k^{2}+A\right) \widehat{u}(k)=\widehat{f}(k)=x / 2$. We have shown that $-k^{2}+A$ is surjective. Hence $k^{2} \in \varrho(A)$.

Now let $f \in C_{\mathrm{per}}^{\alpha}([-\pi, \pi], X)$ be even and let $u \in C^{2+\alpha}([0, \pi], X) \cap$ $C^{\alpha}([0, \pi], D(A))$ be the solution of (4.5). Extend $u$ to an even function; then it is easy to verify that $u \in C_{\text {per }}^{2+\alpha}([-\pi, \pi], X) \cap C_{\text {per }}^{\alpha}([-\pi, \pi], D(A))$ and $u^{\prime \prime}+A u=f$ on $[-\pi, \pi]$. From Lemma 4.3 we have $\left(-k^{2}+A\right) \widehat{u}(k)=\widehat{f}(k)$ $(k \in \mathbb{Z})$. This implies that $\left(u^{\prime \prime}\right)^{\wedge}(k)=k^{2} R\left(k^{2}, A\right) \widehat{f}(k)(k \in \mathbb{Z})$. By Lemma 4.12, $\left(k^{2} R\left(k^{2}, A\right)\right)_{k \in \mathbb{Z}}$ is a $C_{2 \pi}^{\alpha}$-multiplier. It follows from Lemma 3.2 that $\sup _{k \in \mathbb{Z}}\left\|k^{2} R\left(k^{2}, A\right)\right\|<\infty$.

(i) $\Rightarrow$ (ii). Assume that $\sup _{k \in \mathbb{Z}}\left\|k^{2} R\left(k^{2}, A\right)\right\|<\infty$. Then by using an argument similar to the proof of Theorem 4.2 we can show that $\left(k^{2} R\left(k^{2}, A\right)\right)_{k \in \mathbb{Z}}$ is a $C_{2 \pi}^{\alpha}$-multiplier. Let $f \in C^{\alpha}([0, \pi], X)$. Extend $f$ to an even function; then $f \in C_{\text {per }}^{\alpha}([-\pi, \pi], X)$. There exists $v \in C_{\text {per }}^{\alpha}([-\pi, \pi], X)$ such that $\widehat{v}(k)=k^{2} R\left(k^{2}, A\right) \widehat{f}(k) \quad(k \in \mathbb{Z})$. Then $v$ is even and $\widehat{v}(0)=0$. Let $x=$ $\int_{0}^{\pi}\left(s-(2 \pi)^{-1} s^{2}\right) v(s) d s+A^{-1} \widehat{f}(0)$ and let $u(t)=\int_{0}^{t}(t-s) v(s) d s+x$. Then $u \in C_{\text {per }}^{2+\alpha}([-\pi, \pi], X)$ and $u^{\prime}(0)=u^{\prime}(\pi)=0$. An easy computation shows that $\widehat{u}(k)=-R\left(k^{2}, A\right) \widehat{f}(k),\left(u^{\prime \prime}\right)^{\wedge}(k)=k^{2} R\left(k^{2}, A\right) \widehat{f}(k)(k \in \mathbb{Z})$. Now $\left(-k^{2}+A\right) \widehat{u}(k)=\widehat{f}(k)(k \in \mathbb{Z})$ and Lemma 4.3 shows that $u^{\prime \prime}+A u=f$ on $[-\pi, \pi]$. In particular, $u \in C^{\alpha}([-\pi, \pi], D(A))$ and $u^{\prime \prime}+A u=f$ on $[0, \pi]$.

5. The multiplier theorem on the real line. Let $X, Y$ be Banach spaces and let $0<\alpha<1$. In order to define multipliers on this space, it is necessary to operate modulo the constant functions (see [Tr, Sections 5.1.2 and 5.2.2], noting that $C^{\alpha}(\mathbb{R}, \mathbb{C})$ is the homogeneous Besov space $\left.\dot{B}_{\infty, \infty}^{\alpha}(\mathbb{R})\right)$.

The kernel of the seminorm $\|\cdot\|_{\alpha}$ on $C^{\alpha}(\mathbb{R}, X)$ given by $(2.1)$ is the space of all constant functions and the corresponding quotient space $\dot{C}^{\alpha}(\mathbb{R}, X)$ is a Banach space in the induced norm. We will frequently identify a function 
$f \in C^{\alpha}(\mathbb{R}, X)$ with its equivalence class

$$
\dot{f}:=\left\{g \in C^{\alpha}(\mathbb{R}, X): f-g \equiv \text { constant }\right\} .
$$

In particular, when considering $\dot{f}$ we may assume that $f(0)=0$. In this way, $\dot{C}^{\alpha}(\mathbb{R}, X)$ may be identified with the space of all $f \in C^{\alpha}(\mathbb{R}, X)$ with $f(0)=0$.

The next lemma enables us to define multipliers from $\dot{C}^{\alpha}(\mathbb{R}, X)$ into $\dot{C}^{\alpha}(\mathbb{R}, Y)$. It follows from [ABHN, Theorems 4.8.2 and 4.8.1]. By $\mathcal{D}(\mathbb{R} \backslash\{0\})$ we denote the space of all $C^{\infty}$-functions on $\mathbb{R}$ having compact support in $\mathbb{R} \backslash\{0\}$.

Lemma 5.1. Let $f \in C^{\alpha}(\mathbb{R}, X)$. Then

$$
\int_{\mathbb{R}} f(s)(\mathcal{F} \varphi)(s) d s=0 \quad \text { for all } \varphi \in \mathcal{D}(\mathbb{R} \backslash\{0\})
$$

if and only if $f$ is constant.

Definition 5.2. Let $M: \mathbb{R} \backslash\{0\} \rightarrow \mathcal{L}(X, Y)$ be continuous. We say that $M$ is a $\dot{C}^{\alpha}$-multiplier if there exists a mapping $L: \dot{C}^{\alpha}(\mathbb{R}, X) \rightarrow \dot{C}^{\alpha}(\mathbb{R}, Y)$ such that

$$
\int_{\mathbb{R}}(L f)(s)(\mathcal{F} \varphi)(s) d s=\int_{\mathbb{R}}(\mathcal{F}(\varphi M))(s) f(s) d s
$$

for all $f \in C^{\alpha}(\mathbb{R}, X)$ and all $\varphi \in \mathcal{D}(\mathbb{R} \backslash\{0\})$.

Here $(\mathcal{F}(\varphi M))(s)=\int_{\mathbb{R}} e^{-i s t} \varphi(t) M(t) d t \in \mathcal{L}(X, Y)$. Note that the righthand side of (5.1) does not depend on the representative of $\dot{f}$ since

$$
\int_{\mathbb{R}}(\mathcal{F}(\varphi M))(s) d s=2 \pi(\varphi M)(0)=0 .
$$

Moreover, the identity (5.1) defines $L f \in C^{\alpha}(\mathbb{R}, X)$ uniquely up to an additive constant, by Lemma 5.1. Hence, if (5.1) holds, then $L: \dot{C}^{\alpha}(\mathbb{R}, X) \rightarrow$ $\dot{C}^{\alpha}(\mathbb{R}, Y)$ is well defined and linear. The closed graph theorem implies that $L$ is continuous.

Let $\operatorname{sp}_{\mathrm{C}}(f)$ be the Carleman spectrum of $f$, i.e., the support of the distributional Fourier transform of $f$ (see [ABHN, Section 4.8]). It follows from (5.1) that $\operatorname{sp}_{C}(L f) \subset \operatorname{sp}_{C}(f) \cup\{0\}$. Suppose that $0 \notin \operatorname{sp}_{\mathrm{C}}(f)$. Then the theory of the Carleman spectrum shows that there exists a unique $x \in X$ such that $0 \notin \operatorname{sp}_{\mathrm{C}}(\widetilde{L} f)$, where

$$
\widetilde{L} f(t)=L f(t)-x .
$$

Then $\widetilde{L}$ is a well defined linear mapping, satisfying (5.1), on the space of all $f \in C^{\alpha}(\mathbb{R}, X)$ such that $0 \notin \operatorname{sp}_{\mathrm{C}}(f)$.

Our aim is to prove the following multiplier theorem (see [Tr, Section $5.2 .2]$ for a result of this type in the scalar case). We make use of the space $\mathcal{M}(\mathbb{R} \backslash\{0\}, \mathcal{L}(X, Y))$ defined in Section 2. 
Theorem 5.3. Let $M \in \mathcal{M}(\mathbb{R} \backslash\{0\}, \mathcal{L}(X, Y))$. Then $M$ is a $\dot{C}^{\alpha}$-multiplier.

The proof will be similar to that of Theorem 3.4. We start by considering multipliers with compact support for which we have the basic estimate of Proposition 2.1. This corresponds to part (a) of the proof of Theorem 3.4.

Proposition 5.4. Let $M \in C_{\mathrm{c}}^{2}(\mathbb{R} \backslash\{0\}, \mathcal{L}(X, Y))$. Then $M$ is a $\dot{C}^{\alpha}$ multiplier and the associated operator $L$ is given by

$$
L f=\mathcal{F}^{-1} M * f \quad\left(f \in \dot{C}^{\alpha}(\mathbb{R}, X)\right) .
$$

In particular

$$
\|L f\|_{\alpha} \leq \frac{c_{\alpha}}{2 \pi}\|M\|_{\mathcal{M}}\|f\|_{\alpha}
$$

for all $f \in \dot{C}^{\alpha}(\mathbb{R}, X)$, where $c_{\alpha}$ is the constant of Proposition 2.1.

Proof. Let $\varphi \in \mathcal{D}(\mathbb{R} \backslash\{0\})$ and $\psi=\mathcal{F} \varphi \in \mathcal{S}(\mathbb{R})$. For $r \in \mathbb{R}$ let $\psi_{r}(s)=$ $\psi(s+r)$. Then $\left(\mathcal{F}^{-1} \psi_{r}\right)(s)=e^{-i s r} \varphi(s)$. Observe that by Fubini's theorem

$$
\int_{\mathbb{R}}\left(\mathcal{F}^{-1} h\right)(s) k(s) d s=\int_{\mathbb{R}} h(s)\left(\mathcal{F}^{-1} k\right)(s) d s
$$

for all integrable functions $h, k$ on $\mathbb{R}$, one scalar and the other vector-valued. Hence

$$
\begin{aligned}
\int_{\mathbb{R}}\left(\mathcal{F}^{-1} M\right)(s-r) \psi(s) d s & =\int_{\mathbb{R}}\left(\mathcal{F}^{-1} M\right)(s) \psi_{r}(s) d s \\
& =\int_{\mathbb{R}} M(s)\left(\mathcal{F}^{-1} \psi_{r}\right)(s) d s=\int_{\mathbb{R}} M(s) e^{-i s r} \varphi(s) d s \\
& =(\mathcal{F}(\varphi M))(r) .
\end{aligned}
$$

Let $f \in C^{\alpha}(\mathbb{R}, X)$ and $L f=\mathcal{F}^{-1} M * f$. Since $\mathcal{F}^{-1} M=\frac{1}{2 \pi} \mathcal{F} \check{M}$, where $\check{M}(t)=M(-t)$, Proposition 2.1 shows that

$$
L f \in C^{\alpha}(\mathbb{R}, X) \quad \text { and } \quad\|L f\|_{\alpha} \leq \frac{c_{\alpha}}{2 \pi}\|M\|_{\mathcal{M}}\|f\|_{\alpha} .
$$

It follows from (2.4) that

$$
\iint_{\mathbb{R}}\left\|\left(\mathcal{F}^{-1} M\right)(s-r)\right\|\|f(r)\| d r|\psi(s)| d s<\infty .
$$

Fubini's theorem gives

$$
\begin{aligned}
\int_{\mathbb{R}}\left(\mathcal{F}^{-1} M * f\right)(s)(\mathcal{F} \varphi)(s) d s & =\int_{\mathbb{R}} \int_{\mathbb{R}}\left(\mathcal{F}^{-1} M\right)(s-r) f(r) d r \psi(s) d s \\
& =\int_{\mathbb{R}} \int_{\mathbb{R}}\left(\mathcal{F}^{-1} M\right)(s-r) \psi(s) d s f(r) d r \\
& =\int_{\mathbb{R}}(\mathcal{F}(\varphi M))(r) f(r) d r .
\end{aligned}
$$

This shows that $L f:=\mathcal{F}^{-1} M * f$ satisfies (5.1). 
Proof of Theorem 5.3. (a) We first assume that $Y=\mathbb{C}$. Let $M \in$ $\mathcal{M}(\mathbb{R} \backslash\{0\}, \mathcal{L}(X, \mathbb{C}))$. By Corollary 2.3 , there is a sequence $\left(M_{n}\right)_{n \in \mathbb{N}} \subset$ $C_{\mathrm{c}}^{2}(\mathbb{R} \backslash\{0\}, \mathcal{L}(X, \mathbb{C}))$ such that $M_{n}(t) \rightarrow M(t)$ uniformly on compact subsets of $\mathbb{R} \backslash\{0\}$ and $\left\|M_{n}\right\|_{\mathcal{M}} \leq \kappa^{2}\|M\|_{\mathcal{M}}$. Let $f \in C^{\alpha}(\mathbb{R}, X), g_{n}=\mathcal{F}^{-1} M_{n} * f$, $h_{n}=g_{n}-g_{n}(0)$. Then

$$
\left\|h_{n}\right\|_{\alpha}=\left\|g_{n}\right\|_{\alpha} \leq \frac{c_{\alpha} \kappa^{2}}{2 \pi}\|M\|_{\mathcal{M}}\|f\|_{\alpha}=: \bar{c}\|f\|_{\alpha}
$$

By Ascoli's theorem there exists a subsequence $\left(h_{n_{k}}\right)_{k \in \mathbb{N}}$ converging to a function $h: \mathbb{R} \rightarrow \mathbb{C}$ uniformly on compact subsets of $\mathbb{R}$. Then $h \in C^{\alpha}(\mathbb{R}, \mathbb{C})$ and $\|h\|_{\alpha} \leq \bar{c}\|f\|_{\alpha}$. Let $\varphi \in \mathcal{D}(\mathbb{R} \backslash\{0\})$. Since $\mathcal{F} \varphi \in \mathcal{S}(\mathbb{R})$ and $\left|h_{n}(s)\right| \leq$ $\bar{c}\|f\|_{\alpha}|s|^{\alpha}$, we have

$$
\begin{aligned}
\int_{\mathbb{R}} h(s)(\mathcal{F} \varphi)(s) d s & =\lim _{k \rightarrow \infty} \int_{\mathbb{R}} h_{n_{k}}(s)(\mathcal{F} \varphi)(s) d s \\
& =\lim _{k \rightarrow \infty} \int_{\mathbb{R}}\left(\mathcal{F}\left(\varphi M_{n_{k}}\right)\right)(s) f(s) d s \\
& =\int_{\mathbb{R}}(\mathcal{F}(\varphi M))(s) f(s) d s .
\end{aligned}
$$

Thus $L f:=h$ satisfies (5.1).

(b) Now let $Y$ be arbitrary. Let $f \in C^{\alpha}(\mathbb{R}, X)$. For $y^{*} \in Y^{*}$ we can apply (a) to $y^{*} \circ M$. Thus there exists a unique function $g_{y^{*}} \in C^{\alpha}(\mathbb{R}, \mathbb{C})$ such that $g_{y^{*}}(0)=0$ and

$$
\int_{\mathbb{R}} g_{y^{*}}(s)(\mathcal{F} \varphi)(s) d s=\int_{\mathbb{R}}\left(\mathcal{F}\left(\varphi \cdot\left(y^{*} \circ M\right)\right)\right)(s) f(s) d s
$$

for all $\varphi \in \mathcal{D}(\mathbb{R} \backslash\{0\})$; moreover,

$$
\left\|g_{y^{*}}\right\|_{\alpha} \leq \frac{c_{\alpha} \kappa^{2}}{2 \pi}\|M\|_{\mathcal{M}}\|f\|_{\alpha}\left\|y^{*}\right\|
$$

It follows that, for each $t \in \mathbb{R}$, the mapping $y^{*} \mapsto g_{y^{*}}(t)$ is linear and continuous. So we find $g: \mathbb{R} \rightarrow Y^{* *}$ such that $\left\langle g(t), y^{*}\right\rangle=g_{y^{*}}(t)$ for all $y^{*} \in Y^{*}$ and all $t \in \mathbb{R}$. Consequently, $g \in C^{\alpha}\left(\mathbb{R}, Y^{* *}\right)$ and

$$
\|g\|_{\alpha} \leq \frac{c_{\alpha} \kappa^{2}}{2 \pi}\|M\|_{\mathcal{M}}\|f\|_{\alpha}
$$

We have

$$
\begin{aligned}
\left\langle\int_{\mathbb{R}} g(s)(\mathcal{F} \varphi)(s) d s, y^{*}\right\rangle & =\int_{\mathbb{R}}\left(\mathcal{F}\left(\varphi \cdot\left(y^{*} \circ M\right)\right)\right)(s) f(s) d s \\
& =\left\langle y^{*}, \int_{\mathbb{R}}(\mathcal{F}(\varphi M))(s) f(s) d s\right\rangle
\end{aligned}
$$


for all $y^{*} \in Y^{*}$. Identifying $Y$ with a subspace of $Y^{* *}$ we conclude that

$$
\int_{\mathbb{R}} g(s)(\mathcal{F} \varphi)(s) d s=\int_{\mathbb{R}}(\mathcal{F}(\varphi M))(s) f(s) d s
$$

for all $\varphi \in \mathcal{D}(\mathbb{R} \backslash\{0\})$. Consider the quotient mapping $q: Y^{* *} \rightarrow Y^{* *} / Y$. Then $q \circ g \in C^{\alpha}\left(\mathbb{R}, Y^{* *} / Y\right)$ and $(q \circ g)(0)=0$. Since $\int_{\mathbb{R}}(\mathcal{F}(\varphi M))(s) f(s) d s$ $\in Y$, one has

$$
\begin{aligned}
\int_{\mathbb{R}}(q \circ g)(s)(\mathcal{F} \varphi)(s) d s & =q\left(\int_{\mathbb{R}} g(s)(\mathcal{F} \varphi)(s) d s\right) \\
& =q\left(\int_{\mathbb{R}}(\mathcal{F}(\varphi M))(s) f(s) d s\right)=0
\end{aligned}
$$

for all $\varphi \in \mathcal{D}(\mathbb{R} \backslash\{0\})$. By Lemma 5.1 this implies that $(q \circ g)(t)=(q \circ g)(0)=0$ for all $t \in \mathbb{R}$. Hence $g(t) \in Y$ for all $t \in \mathbb{R}$. Thus $L f:=g$ satisfies (5.1).

REMARK 5.5. If $X$ is $B$-convex, Theorem 5.3 remains valid if $\mathcal{M}(\mathbb{R} \backslash\{0\}$, $\mathcal{L}(X, Y))$ is replaced by the space of all $M \in C^{1}(\mathbb{R} \backslash\{0\}, \mathcal{L}(X, Y))$ such that $\|M\|_{\mathcal{M}_{1}}<\infty$. The proof is almost the same as above. In Proposition 5.4, Proposition 2.8 is used instead of Proposition 2.1. In part (a) of the proof of Theorem 5.3, the construction in Corollary 2.3 produces a sequence $\left.\left(M_{n}\right)_{n \in \mathbb{N}} \subset C_{\mathrm{c}}^{1}(\mathbb{R} \backslash\{0\}), \mathcal{L}(X, \mathbb{C})\right)$ approximating $M$. In order to apply Proposition 5.4 , one needs that $M_{n} \in C_{\mathrm{c}}^{2}(\mathbb{R} \backslash\{0\}, \mathcal{L}(X, \mathbb{C}))$. This can be achieved by replacing $M_{n}$ by $\varrho_{n} * M_{n}$ for suitable $\varrho_{n} \in C_{\mathrm{c}}^{2}(\mathbb{R}, \mathbb{C})$.

ExAmPle 5.6 (Riesz projection and Hilbert transform). Let $X$ be an arbitrary Banach space and $0<\alpha<1$.

(a) Let $M(t)=I$ for $t \geq 0$ and $M(t)=0$ for $t<0$. It follows from Theorem 5.3 that $M$ is a $\dot{C}^{\alpha}$-multiplier. The associated operator on $\dot{C}^{\alpha}(\mathbb{R}, X)$ is called the Riesz projection.

(b) Let $M(t)=(-i \operatorname{sign} t) I(t \in \mathbb{R})$. Then $M$ is a $\dot{C}^{\alpha}$-multiplier by Theorem 5.3. The associated operator on $\dot{C}^{\alpha}(\mathbb{R}, X)$ is called the Hilbert transform.

6. Differential equations on the line. Let $A$ be a closed linear operator on $X$ and let $\alpha \in(0,1)$. Given $f \in C^{\alpha}(\mathbb{R}, X)$, we consider the problem

$$
u^{\prime}(t)=A u(t)+f(t) \quad(t \in \mathbb{R}) .
$$

We say that (6.1) is $C^{\alpha}$-well-posed if for each $f \in C^{\alpha}(\mathbb{R}, X)$ there is a unique solution $u \in C^{1+\alpha}(\mathbb{R}, X) \cap C^{\alpha}(\mathbb{R}, D(A))$ of $(6.1)$. Here $D(A)$ is considered as a Banach space with the graph norm, and $C^{1+\alpha}(\mathbb{R}, X)$ is the Banach space of all $u \in C^{1}(\mathbb{R}, X)$ such that $u^{\prime} \in C^{\alpha}(\mathbb{R}, X)$, equipped with the norm

$$
\|u\|_{C^{1+\alpha}}=\left\|u^{\prime}\right\|_{C^{\alpha}}+\|u(0)\| \text {. }
$$


If (6.1) is $C^{\alpha}$-well-posed, it follows from the closed graph theorem that the mapping $L: C^{\alpha}(\mathbb{R}, X) \rightarrow C^{1+\alpha}(\mathbb{R}, X)$ which associates to $f$ the solution $u$ is linear and continuous.

Our aim is to prove the following characterization of $C^{\alpha}$-well-posedness.

TheOrem 6.1. The following assertions are equivalent:

(i) $i \mathbb{R} \subset \varrho(A)$ and $\sup _{s \in \mathbb{R}}\|s R(i s, A)\|<\infty$;

(ii) the problem (6.1) is $C^{\alpha}$-well-posed.

We start with the following lemma. Here we define id : $\mathbb{R} \rightarrow \mathbb{R}$ by $\operatorname{id}(s)=i s$.

Lemma 6.2. Let $0<\alpha<1$ and $u, v \in C^{\alpha}(\mathbb{R}, X)$. The following are equivalent:

(i) $u \in C^{1+\alpha}(\mathbb{R}, X)$ and $u^{\prime}-v$ is constant;

(ii) $\int_{\mathbb{R}} v(s)(\mathcal{F} \psi)(s) d s=\int_{\mathbb{R}} u(s) \mathcal{F}(\mathrm{id} \cdot \psi)(s) d s$ for all $\psi \in \mathcal{D}(\mathbb{R} \backslash\{0\})$.

Proof. (i) $\Rightarrow$ (ii). Let $\psi \in \mathcal{D}(\mathbb{R} \backslash\{0\})$. Then

$$
\begin{aligned}
\int_{\mathbb{R}} v(s)(\mathcal{F} \psi)(s) d s & =\int_{\mathbb{R}} u^{\prime}(s)(\mathcal{F} \psi)(s) d s \\
& =-\int_{\mathbb{R}} u(s)(\mathcal{F} \psi)^{\prime}(s) d s=\int_{\mathbb{R}} u(s) \mathcal{F}(i d \cdot \psi)(s) d s .
\end{aligned}
$$

(ii) $\Rightarrow$ (i). Let $\varphi \in \mathcal{D}(\mathbb{R} \backslash\{0\})$, and $\psi(s)=i \varphi(s) / s$. Then $\psi \in \mathcal{D}(\mathbb{R} \backslash\{0\})$ and $\mathcal{F} \varphi=-\mathcal{F}(\mathrm{id} \cdot \psi)=(\mathcal{F} \psi)^{\prime}$. Let $w(t)=\int_{0}^{t} v(s) d s$. Then integration by parts and the assumption give

$$
\begin{aligned}
\int_{\mathbb{R}} w(s)(\mathcal{F} \varphi)(s) d s & =-\int_{\mathbb{R}} v(s)(\mathcal{F} \psi)(s) d s=-\int_{\mathbb{R}} u(s) \mathcal{F}(\mathrm{id} \cdot \psi)(s) d s \\
& =\int_{\mathbb{R}} u(s)(\mathcal{F} \varphi)(s) d s .
\end{aligned}
$$

It follows from [ABHN, Theorems 4.8.2 and 4.8.1] that $w-u$ is a polynomial. Since $\|w(t)\| \leq c\left(1+|t|^{\alpha+1}\right)$ it follows that $u(t)=w(t)+x+t y=\int_{0}^{t} v(s) d s+$ $x+t y$ for some vectors $x, y \in X$. Thus $u^{\prime}=v+y$.

Proof of Theorem 6.1. (i) $\Rightarrow$ (ii). The argument is similar to Theorem 4.2. Define $M \in C^{2}(\mathbb{R}, \mathcal{L}(X, D(A)))$ by $M(s)=R(i s, A)$. It is easy to see that $M \in \mathcal{M}(\mathbb{R}, \mathcal{L}(X, D(A)))$ and id $\cdot M \in \mathcal{M}(\mathbb{R}, \mathcal{L}(X))$. Let $f \in C^{\alpha}(\mathbb{R}, X)$. By Theorem 5.3 there exist $u \in C^{\alpha}(\mathbb{R}, D(A))$ and $v \in C^{\alpha}(\mathbb{R}, X)$ such that

$$
\begin{aligned}
& \int_{\mathbb{R}} u(s)(\mathcal{F} \varphi)(s) d s=\int_{\mathbb{R}} \mathcal{F}(\varphi \cdot R(i \cdot A))(s) f(s) d s, \\
& \int_{\mathbb{R}} v(s)(\mathcal{F} \psi)(s) d s=\int_{\mathbb{R}} \mathcal{F}(\psi \cdot \mathrm{id} \cdot R(i \cdot A))(s) f(s) d s
\end{aligned}
$$


for all $\varphi, \psi \in \mathcal{D}(\mathbb{R} \backslash\{0\})$. Choosing $\varphi=$ id $\cdot \psi$, it follows from Lemma 6.2 that $u \in C^{1+\alpha}(\mathbb{R}, X)$ and $u^{\prime}=v+y_{1}$ for some $y_{1} \in X$. Since

$$
\mathrm{id} \cdot R(i \cdot A)-A R(i \cdot, A)=I \text {, }
$$

choosing $\varphi=\psi$ gives

$$
\int_{\mathbb{R}} v(s)(\mathcal{F} \psi)(s) d s=\int_{\mathbb{R}} A u(s)(\mathcal{F} \psi)(s) d s+\int_{\mathbb{R}} f(s)(\mathcal{F} \psi)(s) d s
$$

for all $\psi \in \mathcal{D}(\mathbb{R} \backslash\{0\})$. By Lemma 5.1 this implies that for some $y_{2} \in X$ one has

$$
v(t)=A u(t)+f(t)+y_{2} \quad(t \in \mathbb{R}) .
$$

Consequently, $u^{\prime}(t)=A u(t)+f(t)+y(t \in \mathbb{R})$ where $y=y_{1}+y_{2}$. Let $x=A^{-1} y$. Then $u_{1}(t):=u(t)+x$ solves (6.1). We have shown that a solution of (6.1) exists.

In order to prove uniqueness we consider $u \in C^{1+\alpha}(\mathbb{R}, X) \cap C^{\alpha}(\mathbb{R}, D(A))$ such that $u^{\prime}(t)=A u(t)(t \in \mathbb{R})$. We have to show that $u \equiv 0$. Consider the Carleman transform $\widehat{u}$ of $u$ (see [ABHN, (4.25), p. 292]). Since $\widehat{u^{\prime}}(\lambda)=$ $\lambda \widehat{u}(\lambda)-u(0)(\operatorname{Re} \lambda \neq 0)$, one has $\widehat{u}(\lambda) \in D(A)$ and $(\lambda-A) \widehat{u}(\lambda)=u(0)$ for all $\lambda \in \mathbb{C} \backslash i \mathbb{R}$. Since $i \mathbb{R} \subset \varrho(A)$, it follows that the Carleman spectrum $\operatorname{sp}_{\mathrm{C}}(u)$ of $u$ is empty. Hence $u \equiv 0$ by [ABHN, Theorem 4.8.2]. Thus (6.1) is $C^{\alpha}$-well-posed.

(ii) $\Rightarrow\left(\right.$ i). Assume that (6.1) is $C^{\alpha}$-well-posed. Denote by $L: C^{\alpha}(\mathbb{R}, X) \rightarrow$ $C^{1+\alpha}(\mathbb{R}, X)$ the bounded operator which associates to each $f \in C^{\alpha}(\mathbb{R}, X)$ the solution $u$ of (6.1).

Let $\eta \in \mathbb{R}$. We show that $i \eta \in \varrho(A)$. Let $x \in X$ be such that $A x=i \eta x$. Let $u=e_{\eta} \otimes x$, i.e., $u(t)=e^{i \eta t} x$. Then $u^{\prime}(t)=A u(t)$; that is, $u$ is a solution of (6.1) with $f \equiv 0$. Hence $u \equiv 0$, i.e., $x=0$. We have shown that $i \eta-A$ is injective. In order to show surjectivity let $y \in X$. Let $f=e_{\eta} \otimes y$ and $u=L f$. Then for fixed $s \in \mathbb{R}, v_{1}(t)=u(t+s)$ and $v_{2}(t)=e^{i s \eta} u(t)$ are both solutions of $v^{\prime}=A v+e^{i s \eta} f$. Hence $v_{1}=v_{2}$; that is, $u(t+s)=e^{i \eta s} u(t)$ for all $t \in \mathbb{R}, s \in \mathbb{R}$. Let $x=u(0) \in D(A)$. Then $i \eta x=u^{\prime}(0)=A u(0)+f(0)=$ $A x+y$. Hence $(i \eta-A) x=y$. Thus $i \eta-A$ is bijective and so $i \eta \in \varrho(A)$ and $L f=u=e_{\eta} \otimes R(i \eta, A) y$. Consequently,

$$
\begin{aligned}
\gamma_{\alpha}|\eta|^{\alpha}\|\eta R(i \eta, A) y\| & =\left\|i \eta e_{\eta} \otimes R(i \eta, A) y\right\|_{\alpha}=\left\|u^{\prime}\right\|_{\alpha} \leq\|u\|_{C^{1+\alpha}} \\
& \leq\|L\|\|f\|_{C^{\alpha}}=\|L\|\left(\|f\|_{\alpha}+\|f(0)\|\right) \\
& =\|L\|\left(\gamma_{\alpha}|\eta|^{\alpha}\|y\|+\|y\|\right) .
\end{aligned}
$$

Thus $\|\eta R(i \eta, A)\| \leq\|L\|\left(1+\gamma_{\alpha}^{-1}|\eta|^{-\alpha}\right)$. Since $\sup _{|\eta| \leq 1}\|\eta R(i \eta, A)\|<\infty$ by continuity, it follows that (i) holds.

Remarks 6.3. (a) When condition (i) of Theorem 6.1 is satisfied, the functions $M:=R(i \cdot, A)$ and id $\cdot M$ satisfy the respective inhomogeneous Mikhlin conditions (Remark 2.6). It follows from the multiplier theorems of 
[Am], [GW] that if $f \in C^{\alpha}(\mathbb{R}, X)$ is bounded, then the solution $u$ of (6.1) and $u^{\prime}$ are both bounded.

(b) Suppose that $i \mathbb{R} \backslash\{0\} \subset \varrho(A)$ and $\sup _{s \in \mathbb{R} \backslash\{0\}}\|s R(i s, A)\|=: C<\infty$. Then $M(s):=i s R(i s, A)$ defines $M \in \mathcal{M}(\mathbb{R} \backslash\{0\}, \mathcal{L}(X))$. Let $f \in C^{\alpha}(\mathbb{R}, X)$ and $L f$ be as in Definition 5.2. One might hope that there will be a solution $u$ of (6.1) such that $u^{\prime}$ coincides with $L f$ up to a constant, but in general this is not true (cf. Remark 4.7). However the situation is different when $0 \notin \operatorname{sp}_{\mathrm{C}}(f)$. Using the techniques of Theorem 6.1 and the remarks preceding Theorem 5.3, it is then possible to show that (6.1) has a unique solution $u \in C^{1+\alpha}(\mathbb{R}, X) \cap C^{\alpha}(\mathbb{R}, D(A))$ with $0 \notin \operatorname{sp}_{\mathrm{C}}(u)$. Moreover, $\operatorname{sp}_{\mathrm{C}}(u) \subset \operatorname{sp}_{\mathrm{C}}(f)$ and $\left\|u^{\prime}\right\|_{\alpha} \leq c\|f\|_{\alpha}$ for some constant $c$ depending only on $\alpha$ and $C$.

Finally, we show how Theorem 5.3 can be used to recover results about generators of bounded holomorphic semigroups (see [Lu, Theorem 4.4.3]).

ExAmple 6.4. Suppose that $A$ is the generator of a bounded holomorphic semigroup $T$ on $X$, and let $M(s)=A R(i s, A)(s \neq 0)$. Then $M \in$ $\mathcal{M}(\mathbb{R} \backslash\{0\}, \mathcal{L}(X))$, so $M$ is a $\dot{C}^{\alpha}$-multiplier. Let $L$ be the associated operator.

Let $f \in C^{\alpha}\left(\mathbb{R}_{+}, X\right)$ with $f(0)=0$. The convolution $T * f$ is defined on $\mathbb{R}_{+}$, and $(T * f)(t) \in D(A)$ and

$$
A(T * f)(t)=\int_{0}^{t} A T(s)(f(t-s)-f(t)) d s+T(t) f(t)-f(t) \quad(t \geq 0) .
$$

Extend $f$ and $T * f$ to $\mathbb{R}$ by putting them equal to 0 on $(-\infty, 0)$. Then $u:=T * f$ is a solution of (6.1). It is not difficult to show that

$$
\int_{0}^{\infty} A(T * f)(s)(\mathcal{F} \varphi)(s) d s=\int_{0}^{\infty}(\mathcal{F}(\varphi M))(s) f(s) d s
$$

for all $\varphi \in \mathcal{D}(\mathbb{R} \backslash\{0\})$. Since $\|A(T * f)(t)\| \leq c t^{\alpha}(t \geq 0)$, it follows that $L f=A(T * f)$ (see Lemma 5.1). Thus $A(T * f) \in C^{\alpha}\left(\mathbb{R}_{+}, X\right)$.

\section{References}

[Am] H. Amann, Operator-valued Fourier multipliers, vector-valued Besov spaces, and applications, Math. Nachr. 186 (1997), 5-56.

[ABHN] W. Arendt, C. Batty, M. Hieber and F. Neubrander, Vector-valued Laplace Transforms and Cauchy Problems, Birkhäuser, Basel, 2001.

[AB1] W. Arendt and S. Bu, The operator-valued Marcinkiewicz multiplier theorem and maximal regularity, Math. Z. 240 (2002), 311-343.

[AB2] - - - Operator-valued multiplier theorems characterizing Hilbert spaces, J. Austral. Math. Soc., to appear.

[AB3] - - - Tools for maximal regularity, Math. Proc. Cambridge Philos. Soc. 134 (2003), 317-336. 
[CP] Ph. Clément and J. Prüss, An operator-valued transference principle and maximal regularity on vector-valued $L_{p}$-spaces, in: Evolution Equations and Their Applications in Physics and Life Sciences (Bad Herrenalb, 1998), G. Lumer and L. Weis (eds.), Marcel Dekker, New York, 2001, 67-87.

[CPSW] Ph. Clément, B. de Pagter, F. A. Sukochev and M. Witvliet, Schauder decompositions and multiplier theorems, Studia Math. 138 (2000), 135-163.

[GW] M. Girardi and L. Weis, Operator-valued multiplier theorems on Besov spaces, Math. Nachr. 251 (2003), 34-51.

[Hö] L. Hörmander, The Analysis of Linear Partial Differential Operators I, Springer, Berlin, 1983.

[Lu] A. Lunardi, Analytic Semigroups and Optimal Regularity in Parabolic Problems, Birkhäuser, Basel, 1995.

[Mi] A. Mielke, Über maximale $L^{p}$-Regularität für Differentialgleichungen in Banach- und Hilberträumen, Math. Ann. 227 (1987), 121-133.

[Sch] S. Schweiker, Asymptotics, regularity and well-posedness of first- and secondorder differential equations on the line, Ph.D. thesis, Ulm, 2000.

[Tr] H. Triebel, Theory of Function Spaces, Birkhäuser, Basel, 1983.

[We1] L. Weis, Stability theorems for semigroups via multiplier theorems, in: Differential Equations, Asymptotic Analysis, and Mathematical Physics (Potsdam, 1996), Akademie Verlag, Berlin, 1997, 407-411.

[We2] L. Weis, Operator-valued Fourier multiplier theorems and maximal $L_{p}$-regularity, Math. Ann. 319 (2001), 735-758.

Abteilung Angewandte Analysis

St. John's College

Universität Ulm

University of Oxford

89069 Ulm, Germany

E-mail: arendt@mathematik.uni-ulm.de

bu@mathematik.uni-ulm.de

Oxford OX1 3JP, Great Britain

E-mail: charles.batty@st-johns.oxford.ac.uk

Department of Mathematical Science

University of Tsinghua

100084 Beijing, China 